\title{
Investigation of influence of an obstacle on granular flows by virtue of a depth-integrated theory
}

DOI:

10.1016/j.euromechflu.2020.06.014

\section{Document Version}

Accepted author manuscript

Link to publication record in Manchester Research Explorer

\section{Citation for published version (APA):}

Meng, X., Wang, Y., Chiou, M-C., \& Zhou, Y. (2020). Investigation of influence of an obstacle on granular flows by virtue of a depth-integrated theory. European Journal of Mechanics B. Fluids, 84, 334-349.

https://doi.org/10.1016/j.euromechflu.2020.06.014

\section{Published in:}

European Journal of Mechanics B. Fluids

\section{Citing this paper}

Please note that where the full-text provided on Manchester Research Explorer is the Author Accepted Manuscript or Proof version this may differ from the final Published version. If citing, it is advised that you check and use the publisher's definitive version.

\section{General rights}

Copyright and moral rights for the publications made accessible in the Research Explorer are retained by the authors and/or other copyright owners and it is a condition of accessing publications that users recognise and abide by the legal requirements associated with these rights.

\section{Takedown policy}

If you believe that this document breaches copyright please refer to the University of Manchester's Takedown Procedures [http://man.ac.uk/04Y6Bo] or contact uml.scholarlycommunications@manchester.ac.uk providing relevant details, so we can investigate your claim.

\section{OPEN ACCESS}




\author{
Xiannan Meng ${ }^{1}$, Yongqi Wang ${ }^{2 *}$ Min-Ching Chiou ${ }^{3}$, Yunlai Zhou ${ }^{4}$ \\ ${ }^{1}$ School of Mathematics and Manchester centre for Nonlinear Dynamics, University of Manchester, \\ Manchester, UK \\ ${ }^{2}$ Chair of Fluid Dynamics, Department of Mechanical Engineering, Technical University of Darmstadt, \\ Otto-Berndt-Str. 2, 64287 Darmstadt, Germany \\ 3 The company of Arup, Huaihai Plaza 1045, \\ Xuhui district, Shanghai 200031, China \\ ${ }^{4}$ Centre for Offshore Research and Engineering, National University of Singapore, \\ 1 Engineering Drive 2, Singapore 117576, Singapore
}

\title{
Abstract
}

Understanding granular flows past an obstacle is very important to most possibly avoid damage to human properties and infrastructures. The present paper investigates the influence of an obstacle on dry and fluid-saturated granular flows to gain insights into physics behind them. To this end, we extend the existing depth-integrated theory by considering additional effects from the pore fluid pressure and the granular dilatancy. We revisit a largescale experiment to validate the extended theory. The good agreement between numerical results and experimental data reveals that the granular dilatancy plays a crucial role in the mobility and peak depth. Furthermore, we investigate the influence of obstacles on dynamics of dry granular flows by comparing numerical results with experimental data. It is shown that shock waves, dead zones and vacuum (grain-free zone) well observed in the experiments can be captured. Additionally, a fluid-saturated granular flow past the same obstacle is numerically simulated to interpret the role of the interstitial fluid, especially the pore fluid pressure, in the fluid-granular mixture causing distinct dynamic behaviours from those of a dry granular flow. It is also found that the granular dilatancy has a significant influence on the pore fluid pressure which can mitigate the granular friction. This is consistent with many experimental observations. Additionally, it is demonstrated that the pore fluid pressure is prone to elevate the flow depth in front of a cuboid dam (but not in front of a forward-facing tetrahedral wedge), which in turn mitigates the granular friction. The findings are helpful to understand complex behaviours encountered in geophysical flows and industrial processes.

*The corresponding email: wang@fdy.tu-darmstadt.de 
Keywords: Granular flows; fluid-saturated granular flows; fluid-granular mixture model; experimental comparison; granular dilatancy

\section{Introduction}

The influence of an obstacle on granular flows has attracted scientists' and engineers' attentions due to their close relevance to industrial processes and natural hazards. Particularly, obstacles are often constructed to mitigate the impact of granular flows (e.g. snow avalanche, landslides and debris flows) on humans' infrastructures in the context of geophysical flows. The occurrences of natural hazards are becoming more frequent, which makes it pressing to investigate the influence of an obstacle on granular flows.

The last decades have witnessed significant progress in the understanding of the physics of debris flows. Similar to dry granular flows, debris flows are also dominated by granular friction, but they differ from dry granular flows due to the presence of an interstitial liquid. The difference makes them exhibit remarkably different behaviours from their dry counterparts (see Iverson [1997]). Experiments have evidenced that granular media immersed in water and subjected to shear are prone to dilate or contract, which can cause the interstitial liquid being sucked into pores or being pressed (Guazzelli and Pouliquen [2018]). Furthermore, the pore fluid pressure can correspondingly decrease or increase in relation to its original hydrostatic pressure, which would consolidate or mitigate the granular internal friction, see Iverson et al. [2000], Rondon et al. [2011], Wang et al. [2017], and Meng and Wang [2018].

As for the interaction of a debris flow with obstacles, Canelli et al. [2012] analyzed the impact force of a debris flow on rigid and flexible barriers and discussed the possible formulas by which the impact force could be estimated. Song et al. [2017] designed the experiment and estimated the impact force of a debris flow on the obstacle. Choi et al. [2014] employed the Discrete Element Method (DEM) to provide insights into the influence of an array of baffles on the dynamics of debris flows. Albaba et al. [2015] treated a debris flow as a dry medium and discussed the influence of a vertical wall on the dynamics of debris flows by using DEM. Further, Leonardi et al. [2016] applied the combination of DEM and the Lattice-Boltzmann Method (LBM) to investigate the influence of flexible barriers on debris flows. Indeed, experiments and DEMs can provide insights into the influence of obstacles on dynamics of debris flows. However, the financial cost of an experiment is usually formidable, and the DEM suffers from overwhelming computational burden due to its inherent characteristic of tracking each particle which makes it almost impossible to solve real geophysical flows.

Instead, the granular medium can be treated as a continuum, so that classical continuum 
mechanics can be applied. In the continuum approach, a rheological relation is required to complement the momentum balance equation. The last decade has witnessed success of the $\mu(I)$-rheology (Jop et al. [2006] and Forterre and Pouliquen [2008]) to describe the granular constitutive relation. However, a recent work (Barker et al. [2015]) demonstrates that the $\mu(I)$ rheology suffers from ill-posed behavior in the quasi-static regime, which implies that it cannot be applied to investigate granular flows past an obstacle. A reliable and mature approach to circumvent this difficulty is to create a depth-integrated model by proposing a reasonable friction boundary condition on the bottom and then utilizing depth-integration techniques to transform the mass and momentum balance equations into depth-integrated forms, see Savage and Hutter [1989], Gray et al. [1999], Gray and Edwards [2014], Iverson and George [2014], Meng and Wang [2018] etc. Perhaps only the models of Iverson and George [2014], Bouchut et al. [2016] and Meng and Wang [2018], among the existing depth-integrated models of debris flows (Iverson and Denlinger [2001], Pitman and Le [2005], Pudasaini [2012], Iverson and George [2014], Meng and Wang [2016], Bouchut et al. [2016], Meng and Wang [2017] and Meng and Wang [2018]), incorporate sufficient physics to describe the coupling of the granular dilatancy and the pore fluid pressure, which has a significant influence on the mobility of debris flows and plays a crucial role in hysterical behaviours of such flows (Iverson et al. [2000]; Rondon et al. [2011]). However, these depth-integrated models that take the granular dilatancy into account are formulated either in Cartesian coordinates or simple curvilinear coordinates, such that they principally work for debris flows over a flat topography.

The present paper aims to provide insights into the influence of an obstacle on dry granular and debris flows by employing a depth-integrated model. To this end, we employ the model of Meng and Wang [2018] and consider an obstacle as a basal elevation from the reference plane which follows the landscape topography. The resulting theory takes the granular dilatancy, also the pore fluid pressure for fluid-granular mixture, into account. To validate the theory we revisit a large-scale experiment conducted in USGS flume to mimic debris flow's behaviours. Furthermore, we investigate the influence of an obstacle on dry granular flows, towards the goal to understand the physics behind dry granular flows past an obstacle, though many relevant research have been conducted, see Chu et al. [1995], Faug et al. [2002], Gray et al. [2003], Moriguchi et al. [2009], Kuo et al. [2015], etc. However, most aforementioned studies are confined to ideal configurations, e.g. a uniform granular flow past an obstacle or a two-dimensional problem. Instead, we investigate the granular flow of a finite volume past a sharp and a blunt obstacle, respectively, in which the obstacle is treated as part of the basal topography that consists of an incline, a horizontal runout plane and a smooth transition between them. This configuration 
is more close to real flows compared to those of the aforementioned studies. Additionally, the current theory is applied to investigate fluid-saturated granular flows past the same obstacles and compare the corresponding numerical results with those of dry granular flows to explore the influence of the interstitial fluid (especially the influence of the pore fluid pressure), which to the best of our knowledge has been studied only by Kattel et al. [2018] by employing a depthintegrated theory. Nevertheless, the model of Kattel et al. [2018] does not take the granular dilatancy into account; hence the role of the pore fluid pressure cannot be studied.

\section{Model equations and numerical technique}

\subsection{Governing equations and constitutive model}

A saturated grain-fluid mixture is considered, in which the interstitial liquid fills all the voids between the grains. Principally, there exist two kinds of approaches to describe such flows. Mixture theory is either employed (see Truesdell [1992]) or averaging theory is adopted (see Anderson and Jackson [1967]). These two approaches have different momentum equations for the constituents, but they share the same conservation laws for the mixture as a whole. The mass and momentum conservation equations of the mixture as a whole are given by

$$
\begin{aligned}
& \frac{\partial \rho}{\partial t}+\nabla \cdot(\rho \boldsymbol{u})=0, \\
& \frac{\partial(\rho \boldsymbol{u})}{\partial t}+\nabla \cdot(\rho \boldsymbol{u} \otimes \boldsymbol{u})=\nabla \cdot\left(\boldsymbol{T}_{s}+\boldsymbol{T}_{f}+\boldsymbol{T}^{\prime}\right)+\rho \mathbf{g},
\end{aligned}
$$

where $t$ denotes the time, $\rho$ the mixture density, and $\boldsymbol{u}$ the mixture velocity. The mixture density and velocity are defined by

$$
\rho=\rho_{s}+\rho_{f} \quad \text { and } \quad \boldsymbol{u}=\left(\rho_{s} \boldsymbol{u}_{s}+\rho_{f} \boldsymbol{u}_{f}\right) / \rho,
$$

where $\rho_{s}$ and $\rho_{f}$ are called partial densities in mixture theory. They are connected with the material intrinsic densities $\widetilde{\rho}_{\eta}(\eta=\{s, f\})$ through relation $\rho_{\eta}=\widetilde{\rho}_{\eta} \phi_{\eta}$, in which the quantity $\phi_{\eta}$ is called volume fraction of the $\eta$ constituent, and $\phi_{s}+\phi_{f}=1$ holds for saturated media. $\boldsymbol{u}_{s}$ and $\boldsymbol{u}_{f}$ indicate the solid and the fluid partial velocities, respectively. The mixture velocity $\boldsymbol{u}$ is referred to the barycentre rather than the centre of the volume.

The variable $\boldsymbol{T}_{\eta}(\eta=\{s, f\})$ represents the partial stress tensor of constituent $\eta$. Particularly, the solid partial stress $\boldsymbol{T}_{s}$ can be actually connected with the solid effective stress $\boldsymbol{T}_{e}$ from soil mechanics by $\boldsymbol{T}_{s}=-\phi_{s} p_{f} \mathbf{I}-\boldsymbol{T}_{e}$ (Iverson [1997]). The term $-\phi_{s} p_{f} \mathbf{I}$ denotes the contribution of the pore fluid pressure and the solid effective stress $\boldsymbol{T}_{e}$ represents contact force of grains, in which the emergence of a negative sign in front of $\boldsymbol{T}_{e}$ is due to the convention that 
the compressive stress is positive in soil mechanics. The solid effective stress $\boldsymbol{T}_{e}$ is assumed to satisfy the Mohr-Coulomb yield criterion, already applied by Savage and Hutter [1989]; it implies that the shear stress is proportional to the normal stress by a coefficient as the material yields. The internal shear stress $\boldsymbol{S}=\boldsymbol{T}_{e} \cdot \boldsymbol{n}-\left(\boldsymbol{n} \cdot \boldsymbol{T}_{e} \cdot \boldsymbol{n}\right) \boldsymbol{n}$ and the normal stress $N=\boldsymbol{n} \cdot \boldsymbol{T}_{e} \cdot \boldsymbol{n}$ are related by

$$
|\boldsymbol{S}|=N \tan (\varphi+\psi),
$$

where $\varphi$ is the internal friction angle which can be measured directly and $\psi$ represents the granular dilatancy angle which is determined by a dilatancy law presented in Sect. 2.2. Relation (4) reproduces well-observed behaviors in soil mechanics and granular physics that the shear stress is augmented when the grains are being dilated $(\psi>0)$, but decreased when the grains are compressed $(\psi<0)$.

Additionally, the fluid partial stress is given by $\boldsymbol{T}_{f}=-\phi_{f} p_{f} \mathbf{I}+\phi_{f} \boldsymbol{\tau}_{f}$, and Newtonian behaviour is postulated so that $\boldsymbol{\tau}_{f}=2 \mu_{f} \mathbf{D}$, where $\mu_{f}$ is the fluid dynamic viscosity and $\mathbf{D}=$ $\left(\nabla \mathbf{u}+(\nabla \mathbf{u})^{T}\right) / 2$ is the rate of strain tensor. The stress tensor $\boldsymbol{T}^{\prime}$ in (2) characterizes the contribution of the motions of the solid and fluid constituents relative to the mixture as a whole, defined by

$$
\boldsymbol{T}^{\prime}=\rho_{s} \phi_{s}\left(\boldsymbol{u}_{s}-\boldsymbol{u}\right) \otimes\left(\boldsymbol{u}_{s}-\boldsymbol{u}\right)+\rho_{f} \phi_{f}\left(\boldsymbol{u}_{f}-\boldsymbol{u}\right) \otimes\left(\boldsymbol{u}_{f}-\boldsymbol{u}\right)
$$

where the terms on the right-hand side express the momentum fluxes of the solid and fluid, respectively, relative to the mixture velocity.

The summation of the aforementioned stress tensors leads to $\boldsymbol{T}_{s}+\boldsymbol{T}_{f}+\boldsymbol{T}^{\prime}=-p_{f} \mathbf{I}+\phi_{f} \boldsymbol{\tau}_{f}-$ $\boldsymbol{T}_{e}+\boldsymbol{T}^{\prime}$, which reduces to $-p_{f} \mathbf{I}-\boldsymbol{T}_{e}$ in hydrostatic states. The absence of the fluid volume fraction in the bulk stress in hydrostatic states agrees with the observation from experimental measurements in soil mechanics that the manometric pressure in the soil is the pressure as if the medium were a bulk fluid, unaffected by the presence of the solid constituent in the medium. Further, it is vital to note that the momentum equation (2) can be reduced to a hydrostatic balance. Provided that the solid has the same density as the interstitial fluid, i.e. $\widetilde{\rho}_{s}=\widetilde{\rho}_{f}$, grains would suspend in the fluid and the contact force among grains will vanish, which then implies $\boldsymbol{T}_{e}=0$. In this case, (2) reduces to

$$
\nabla p_{f}=\widetilde{\rho}_{f} \boldsymbol{g}
$$

which exactly describes a hydrostatic balance.

To simplify the model but still capture the key physics, we postulate that the fluid and the solid move with the same bed-aligned velocity, yet the components of the velocities perpendicular 
to the bed are different due to the effect of the granular dilatancy. The physical basis of this postulation lies in the fact that the typical geophysical data imply $\left|\boldsymbol{u}_{f}-\boldsymbol{u}_{s}\right| /\left|\boldsymbol{u}_{s}\right| \ll 1$ (see Iverson and Denlinger [2001] and Iverson and George [2014]). Consequently, it is reasonable to deduce that the fluid and the solid phase move with the same bed-aligned velocity. However, we retain the difference of the components of velocities perpendicular to the bed herein, given that a very small difference can cause the development of a significant excess pore fluid pressure which in turn affects the movement of the grains.

\subsection{Dilatancy law}

A number of experiments (see Guazzelli and Pouliquen [2018]) demonstrate that the granular dilatancy can induce a relative movement between the fluid and the solid, which is linked with the development of excess pore fluid pressure. The original dilatancy law proposed by Roux and Radjai [1998] is used for the description of quasi-static dry granular flows. Pailha and Pouliquen [2009] modified this formulation for granular flows immersed in the water, and the modified form is given by

$$
\begin{aligned}
& -\frac{1}{\phi_{s}} \frac{d \phi_{s}}{d t}=\nabla \cdot \boldsymbol{u}_{s}=\dot{\gamma} \tan \psi, \\
& \tan \psi=k_{1}\left(\phi_{s}-\phi_{e q}\right), \\
& \phi_{e q}=\phi_{c}-k_{2} I_{v},
\end{aligned}
$$

where $\dot{\gamma}$ is a scalar measure of shear rate and $\dot{\gamma} / 2$ represents the square root of the second principal invariant of the granular deviatoric deformation-rate tensor. The parameters $k_{1}$ and $k_{2}$ are positive, $\phi_{e q}$ is the equilibrium solid volume fraction, and $\phi_{c}$ is the critical solid volume fraction observed when a continuous quasi-static deformation takes place. Usually, $\phi_{c}$ determines whether the initial packing is dense $\left(\phi_{s}>\phi_{c}\right)$ or loose $\left(\phi_{s}<\phi_{c}\right)$. The viscous number $I_{v}=$ $\mu_{f} \dot{\gamma} / T_{e(z z)}$ represents the timescale ratio between the grain-rearrangement timescale $\left(\mu_{f} / T_{e(z z)}\right)$ and characteristic time $(1 / \dot{\gamma})$ for bulk shear deformation. Relations (7)-(9) imply that the granular material, subject to shear, will evolve towards the same steady state, no matter whether the initial preparation is loose or dense.

\subsection{Boundary Conditions}

As the granular dilatancy takes place, grains are prone to protrude the water surface. Conversely, grains deposit underneath the water surface in the presence of granular compression. The relative movement in the normal direction poses a challenge to properly define the upper boundary. We follow Iverson and George [2014] to introduce a virtual surface as the upper surface, beneath 
which the mixture mass per unit basal area is the same as the mass between the bottom and top surface, and the volume fraction can be also reasonably assumed to be uniform along the depth direction. It implies that some combination of solid or fluid mass immediately above or below the virtual surface will be replaced by an equivalently massive and homogeneous layer with density $\rho$ and the upper surface at $z=s(x, y, t)$. The above description implies that a material condition

$$
\frac{\partial \mathcal{F}^{(s)}}{\partial t}+\boldsymbol{u}^{(s)} \cdot \nabla \mathcal{F}^{(s)}=0,
$$

holds for the bulk, where the free surface is $\mathcal{F}^{(s)}=z-s(x, y, t)=0$ and the normal vector of the free surface is $\boldsymbol{n}^{(s)}=\nabla \mathcal{F}^{(s)} /\left|\nabla \mathcal{F}^{(s)}\right|$, and the superscript "(s)" identifies the quantities evaluated on the free surface. Additionally, the traction-free condition on the free surface is stipulated for the bulk, which implies

$$
\left(\boldsymbol{T}_{s}^{(s)}+\boldsymbol{T}_{f}^{(s)}\right) \cdot \boldsymbol{n}^{(s)}=\mathbf{0}, \quad z=s(x, y, t) .
$$

On the bottom $\mathcal{F}^{(b)}(x, y, z)=z-b(x, y)=0$, the non-penetration boundary condition is prescribed for the bulk, which implies

$$
\boldsymbol{u}^{(b)} \cdot \boldsymbol{n}^{(b)}=0, \quad \boldsymbol{n}^{(b)}=\nabla \mathcal{F}^{(b)} /\left|\nabla \mathcal{F}^{(b)}\right| .
$$

Additionally, the Coulomb bottom friction condition for the granular phase and Navier slip bottom boundary condition for the fluid phase are adopted,

$$
\begin{aligned}
& \boldsymbol{T}_{e}^{(b)} \boldsymbol{n}^{(b)}-\left(\boldsymbol{n}^{(b)} \cdot \boldsymbol{T}_{e}^{(b)} \boldsymbol{n}^{(b)}\right) \boldsymbol{n}^{(b)}=-\frac{\boldsymbol{u}_{s}^{(b)}}{\left|\boldsymbol{u}_{s}^{(b)}\right|}\left(\boldsymbol{n}^{(b)} \cdot \boldsymbol{T}_{e}^{(b)} \boldsymbol{n}^{(b)}\right) \mu_{s}, \\
& \boldsymbol{T}_{f}^{(b)} \boldsymbol{n}^{(b)}-\left(\boldsymbol{n}^{(b)} \cdot \boldsymbol{T}_{f}^{(b)} \boldsymbol{n}^{(b)}\right) \boldsymbol{n}^{(b)}=k_{f}^{b} \phi_{f} \boldsymbol{u}_{f}^{(b)},
\end{aligned}
$$

which implicitly indicate that there are slip velocities on the bottom. The superscript "(b)" identifies the quantities at the bottom. The reason to specify Coulomb friction on the bottom lies in the fact that a number of evidences indicate that Coulomb friction generates most of the resistance force to debris flows (see Iverson [2003] etc.). In relation (13), the friction coefficient $\mu_{s}=\tan (\delta+\psi)$ incorporates both the classical Coulomb friction coefficient (constant-volume friction angle $\delta$ ) and the influence of the granular dilatancy, which is consistent with (4). Alternatively, one can follow the progress of dry granular flows to employ the friction coefficient proposed by Pouliquen and Forterre [2002], in which the friction coefficient is a function of the Froude number. In (14), $k_{f}^{b}$ characterizes the fluid bed frictional coefficient.

\subsection{Model equations for fluid-saturated granular flows}

The geometric characteristic of geophysical flows, i.e. typical flow thickness much smaller than typical flow length, allows to derive a set of tractable depth-integrated equations. In Meng 
and Wang [2018], a set of depth-integrated equations have been derived. However, a simple curvilinear coordinate system was employed by Meng and Wang [2018], so that the model is mainly valid for debris flows past the topography without any bump. It leads to limited application. We follow Gray et al. [1999] and Meng and Wang [2016] to introduce a quasi-twodimensional reference surface (see Fig. 1) which follows the mean down-slope chute topography. The $x$-axis is oriented in the down-slope direction, the $y$-axis follows the cross-slope direction, and the $z$-axis is normal to them. The down-slope inclination angle $\zeta$ varies as a function of the down-slope coordinate $x$, and there is no lateral variation in the $y$-direction. A complex shallow three-dimensional basal topography is overlapped on the reference surface by an elevation $b(x, y)$. The complete sketch of the coordinate system is demonstrated in Fig. 1.

To proceed, the normal component of the momentum equation (2) can be simplified to derive the solid effective normal stress and the pore fluid pressure by virtue of a thin-layer assumption. Subsequently, the integration of (1) and (2) in the depth direction is required for the purpose to derive depth-integrated equations, which involves lengthy mathematical derivation. For the sake of brevity, the above process is demonstrated in Appendices A and B, and we only provide the derived model equations and the physical interpretation here instead. The derived model equations are given by

$$
\begin{aligned}
& \frac{\partial}{\partial t}(h \bar{\rho})+\frac{\partial}{\partial x}(h \bar{\rho} \bar{u})+\frac{\partial}{\partial y}(h \bar{\rho} \bar{v})=0, \\
& \frac{\partial}{\partial t}\left(h \bar{\rho}_{s}\right)+\frac{\partial}{\partial x}\left(h \bar{\rho}_{s} \bar{u}\right)+\frac{\partial}{\partial y}\left(h \bar{\rho}_{s} \bar{v}\right)=s_{s}, \\
& \frac{\partial}{\partial t}(h \bar{\rho} \bar{u})+\frac{\partial}{\partial x}\left(h \bar{\rho} \bar{u} \bar{u}+\frac{1}{2} \bar{\rho} g h^{2} \cos \zeta\right)+\frac{\partial}{\partial y}(h \bar{\rho} \bar{u} \bar{v})=s_{x}, \\
& \frac{\partial}{\partial t}(h \bar{\rho} \bar{v})+\frac{\partial}{\partial x}(h \bar{\rho} \bar{u} \bar{v})+\frac{\partial}{\partial y}\left(h \bar{\rho} \bar{v} \bar{v}+\frac{1}{2} \bar{\rho} g h^{2} \cos \zeta\right)=s_{y} .
\end{aligned}
$$

where $h$ represents the flow height, $\bar{u}$ the depth-averaged mixture velocity in the down-slope direction (the symbol overbar represents depth-averaged quantity), $\bar{v}$ the depth-averaged mixture velocity in the cross-slope direction, $\bar{\rho}=\bar{\rho}_{f}+\bar{\rho}_{s}$ is the mixture density with $\bar{\rho}_{\eta}=\widetilde{\rho}_{\eta} \bar{\phi}_{\eta}(\eta=$ $\{s, f\})$. The depth-averaged form of an arbitrary quantity $\bar{f}$ is defined by

$$
\bar{f}=\frac{1}{h} \int_{b}^{s} f d z
$$

Equation (15) describes the mass conservation of the mixture, while the solid mass equation (16) describes that the granular mass is not conservative within the mixture. This is due to the fact that in the presence of the granular dilatancy an amount of the granular mass may protrude the water surface. Equations (17) and (18) account for the momentum conservation of the mixture in the $x$ and $y$ directions, respectively. The local time rate of change of the mixture momenta is 
balanced by the convective fluxes on the left-hand sides of eqs. (17) and (18) and various forces on the right-hand sides listed in the source terms as follows,

$$
\begin{aligned}
s_{s}= & -\frac{\bar{\rho}_{s} \widetilde{\rho}_{f}}{\bar{\rho}} 3 \bar{u} \tan \psi_{b}, \\
s_{x}= & \bar{\rho} g h \sin \zeta-\frac{\bar{u}}{\sqrt{\bar{u}^{2}+\bar{v}^{2}}} \mu_{s}\left(\bar{\rho} g h \cos \zeta-p_{b e d}+\left(\widetilde{\rho}_{s}-\widetilde{\rho}_{f}\right) \kappa h \bar{\phi}_{s} \bar{u}^{2}\right) \\
& -k_{f}^{b} \bar{\phi}_{f} \bar{u}-\bar{\rho} g h(\cos \zeta) \frac{\partial b}{\partial x}, \\
s_{y}= & -\frac{\bar{v}}{\sqrt{\bar{u}^{2}+\bar{v}^{2}}} \mu_{s}\left(\bar{\rho} g h \cos \zeta-p_{b e d}+\left(\widetilde{\rho}_{s}-\widetilde{\rho}_{f}\right) \kappa h \bar{\phi}_{s} \bar{u}^{2}\right) \\
& -k_{f}^{b} \bar{\phi}_{f} \bar{v}-\bar{\rho} g h(\cos \zeta) \frac{\partial b}{\partial y},
\end{aligned}
$$

where $p_{\text {bed }}$ represents the basal pore fluid pressure, $\kappa$ the curvature of the basal topography, and $\dot{\gamma}_{b}=3 \sqrt{\bar{u}^{2}+\bar{v}^{2}} / h$. It is noted that the topographic terms $-\bar{\rho} g h(\cos \zeta) \partial b / \partial x$ and $-\bar{\rho} g h(\cos \zeta) \partial b / \partial y$, representing the influence of the basal elevation $b(x, y)$, do not appear in the model of Meng and Wang [2018]. In the present paper, the topographic terms characterize the influence of the obstacle on flow dynamics, and hence they are not trivial.

The source term $s_{s}$ in $(20)$ reflects implicitly that the solid particles protrude the virtual surface due to the granular dilatancy. The source terms $s_{x}$ and $s_{y}$ in (21) and (22) characterize contributions of the gravitational components, the bed Coulomb friction, the bed viscous friction, and the basal topographic elevation term, consecutively. Relation (23) expresses that the granular dilatancy is described by the difference of the solid volume fraction and the equilibrium volume fraction $\phi_{e q}$. The equilibrium volume fraction is a monotonically decreasing function with the increase of shear rate $\dot{\gamma}_{b}$. At zero shear rate $\dot{\gamma}_{b}=0, \phi_{e q}$ equals the critical volume fraction $\phi_{c}$ that differentiates the initially loose packing $\left(\phi_{s}<\phi_{c}\right)$ or dense packing $\left(\phi_{s}>\phi_{c}\right)$. Relations (24) specify the bed pore fluid pressure and the bed Coulomb friction coefficient. The pore fluid pressure includes a hydrostatic and an excess pressure, in which the excess pore fluid pressure $p_{e}=-\mu_{f} h^{2} \dot{\gamma}_{b} \tan \psi_{b} /(2 k)$ is linked with the granular dilatancy. The inclusion of the dilatancy angle in the solid Coulomb friction coefficient $\mu_{s}$ represents the effect of the grains' microscopic arrangement on the macroscopic friction. Relation (23) together with (24) describes that an initially dense packed granular material $\left(\phi_{s}>\phi_{c}\right)$, subject to shear, will dilate and the ambient liquid is therefore sucked into void between grains. It creates an inward flow through the granular skeleton and the pore fluid pressure drops correspondingly from original hydrostatic value. The decrease of $p_{\text {bed }}$ causes that the granular friction is enhanced and the mobility weakens. A contrary behavior occurs for an initially dense packed granular material subject to 
shear.

\subsection{Reduced model equations for dry granular flows}

When the interstitial liquid and the granular dilatancy effects are removed from eqs. (15)-(18), one can derive Savage-Hutter type PDEs (Gray et al. [1999]) which have been extensively proved to be capable to describe dry granular flows. It is the case that, when the fluid volume fraction, the fluid density and the granular dilatancy angle vanish, equations (15)-(18) reduce to

$$
\begin{aligned}
& \frac{\partial h}{\partial t}+\frac{\partial}{\partial x}(h \bar{u})+\frac{\partial}{\partial y}(h \bar{v})=0 \\
& \frac{\partial}{\partial t}(h \bar{u})+\frac{\partial}{\partial x}\left(h \bar{u} \bar{u}+\frac{1}{2} g h^{2} \cos \zeta\right)+\frac{\partial}{\partial y}(h \bar{u} \bar{v})=s_{x} \\
& \frac{\partial}{\partial t}(h \bar{v})+\frac{\partial}{\partial x}(h \bar{u} \bar{v})+\frac{\partial}{\partial y}\left(h \bar{v} \bar{v}+\frac{1}{2} g h^{2} \cos \zeta\right)=s_{y}
\end{aligned}
$$

where the source terms are given by

$$
\begin{aligned}
& s_{x}=h g \sin \zeta-\frac{\bar{u}}{\sqrt{\bar{u}^{2}+\bar{v}^{2}}} h \tan \delta\left(g \cos \zeta+\kappa \bar{u}^{2}\right)-h g(\cos \zeta) \frac{\partial b}{\partial x}, \\
& s_{y}=-\frac{\bar{v}}{\sqrt{\bar{u}^{2}+\bar{v}^{2}}} h \tan \delta\left(g \cos \zeta+\kappa \bar{u}^{2}\right)-h g(\cos \zeta) \frac{\partial b}{\partial y} .
\end{aligned}
$$

\subsection{Numerical method}

Equations (15)-(18) (or eqs. (25)-(27)), complemented by relations (20)-(22) ((28) and (29)), constitute a convection-dominated PDE system. Such a PDE system is hyperbolic and allows the development of shock waves for granular flows down an inclined plane merging into a horizontal runout zone or encountering obstacles when the flow changes from a supercritical state into a subcritical state. Consequently, a robust numerical scheme must be applied to avoid possible numerical oscillations. Many numerical schemes have been applied successfully to identify shock waves in granular flows, e.g. Denlinger and Iverson [2001], Wang et al. [2004], George [2008], Meng and Wang [2016] etc. It is worth mentioning that the NT scheme of Nessyahu and Tadmor [1990], a shock-capturing scheme which does not need to solve Riemann problems, is popular to be used to identify shock waves in granular flows. The NT scheme requires that the model equations (15)-(18) are rewritten in the following vector form

$$
\frac{\partial \mathbf{U}}{\partial t}+\frac{\partial \mathbf{F}}{\partial x}+\frac{\partial \mathbf{G}}{\partial y}=\mathbf{S}
$$


The vector of conservative variables $\mathbf{U}$, the flux vectors $\mathbf{F}$ and $\mathbf{G}$, and the source vector $\mathbf{S}$ are given, respectively, by

$$
\mathbf{U}=\left[\begin{array}{c}
h \bar{\rho} \\
h \bar{\rho}_{s} \\
h \bar{\rho} \bar{u} \\
h \bar{\rho} \bar{v}
\end{array}\right], \mathbf{F}=\left[\begin{array}{c}
h \bar{\rho} \bar{u} \\
h \bar{\rho}_{s} \bar{u} \\
h \bar{\rho} \bar{u}^{2}+\bar{\rho} g h^{2}(\cos \zeta) / 2 \\
h \bar{\rho} \bar{u} \bar{v}
\end{array}\right], \mathbf{G}=\left[\begin{array}{c}
h \bar{\rho} \bar{v} \\
h \bar{\rho}_{s} \bar{v} \\
h \bar{\rho} \bar{u} \bar{v} \\
h \bar{\rho} \bar{v}^{2}+\bar{\rho} g h^{2}(\cos \zeta) / 2
\end{array}\right], \mathbf{S}=\left[\begin{array}{c}
0 \\
s_{s} \\
s_{x} \\
s_{y}
\end{array}\right] .
$$

In the following we will not address how to apply the NT scheme to numerically solve vector equation (30), since Tai et al. [2001] and Wang et al. [2004] have already described details to numerically solve hyperbolic governing equations whose mathematical structure is similar to that of eq. (30) with flux vectors defined by (31).

\section{A debris flow down an inclined plane}

To validate the model equations (15)-(18) a large-scale experiment conducted at the USGS debris-flow flume is revisited. In this large-scale experiment presented in Iverson et al. [2010], a sand-gravel-mud mixture initially distributed behind a gate with an initial geometry shown in Fig. 2 was suddenly released as the gate was opened. Then, the mass accelerated down the chute until it approached a horizontal run-out plane. The transverse dimension of the chute is sufficiently wide that the flow across the transverse section can be considered as uniform. The experimental and computational parameters used to validate the model are presented in Table 1. Because shear causes a higher permeability when the material is moving, we employ a bigger granular permeability than its initial value. In the computation, a domain $x \in[-10,90]$ is employed and it is discretized into 1000 grids with a cell size of $\Delta x=0.1 \mathrm{~m}$.

Figure 3 compares the measured time series of the depth at $x=32 \mathrm{~m}$ and $x=66 \mathrm{~m}$ downslope from the gate with the corresponding numerical results. The comparison shows that the current model can provide a reasonable prediction for the time series of depth profile and the predicted peak depth can match the experimental one well. In Fig. 3 the numerical results with and without the consideration of the granular dilatancy are also compared, in which the solid lines represent the results obtained by considering the granular dilatancy, while the dashed lines denote the results without the granular dilatancy. It clearly demonstrates that considering the granular dilatancy can better predict the peak depth and the mobility of the flow front. This is due to the fact that an initially loosely packed granular material, as used here, will evolve subject to shear towards a consolidate state. It will induce a positive excess pore fluid pressure which can mitigate the granular friction. However, if the granular dilatancy would not be accounted in the modeling, the pore fluid pressure would remain always hydrostatic, which causes that the 
granular friction is over-estimated and hence the flow mobility is under-estimated.

\section{Granular flows past an obstacle}

The investigation of a large-scale flow shown above has demonstrated that the present model can predict the dynamics of a debris flow reasonably well. Here, we apply this model to investigate dry and fluid-saturated granular flows past an obstacle towards the goal to gain insights into the physical mechanism behind the interaction of granular materials and an obstacle. The model results of the dry granular flow will be compared with the experimental data.

\subsection{Experimental set-up}

Experiments of a lump of a dry granular material impinging an obstacle have been performed in Darmstadt. The experiments used Vestolen particles and yellow sand whose material properties are presented in Table 2. In the experiments, two different granular materials, a mass of 1.41 $\mathrm{kg}$ Vestolen particles with density $639 \mathrm{~kg} / \mathrm{m}^{3}$ and a mass of $3.75 \mathrm{~kg}$ yellow sands with density $1661 \mathrm{~kg} / \mathrm{m}^{3}$, respectively, were used. The granular mass was initially held within a shallow cap that can suddenly be opened by pulling a rope connected to a bar above the cap. The initial height profile is given by

$$
h=\sqrt{R^{2}-\left(x-x_{0}\right)^{2}-\left(y-y_{0}\right)^{2}}-h_{0},
$$

where $x_{0}=313.5 \mathrm{~mm}, y_{0}=0, R=238 \mathrm{~mm}$ and $h_{0}=178 \mathrm{~mm}$. Once the cap is removed, the granular materials will accelerate on a chute made of plexiglass with $2 \mathrm{~mm}$ thickness and then flow past a cuboid dam and a forward-facing tetrahedral wedge, respectively. The chute is comprised of an inclined part, a horizontal part and a smooth transition between them. The upper plane is inclined $\zeta=40^{\circ}$ and spans $933.5 \mathrm{~mm}$ downslope. The transition zone spans $146.5 \mathrm{~mm}$ and the horizontal plane spans $835 \mathrm{~mm}$ down-slope. In the cross-slope direction the whole chute spans $1100 \mathrm{~mm}$. Additionally, an electric clock is placed in the upper right part of the chute to identify the time in each frame. The Particle Image Velocimetry (PIV) technique, consisting of a CCD camera and two flashes, is employed in the experiment. The PIV technique can not only capture the geometry of the avalanche flow at each time frame, but also evaluate quantitatively the complex instantaneous velocity field. Detailed explanation for the PIV technique to deduce the velocity field can be found in Pudasaini et al. [2005]. The measurement set-up also includes a digital video camera as a substitute for the synchronizer to record the image during the entire granular motion. Plan view of the experimental set-up is shown in Fig. 4 and more details of the experiments are well documented in the $\mathrm{PhD}$ thesis of 
Chiou [2006]. All experiments have been performed in the laboratory at the Technical University of Darmstadt.

\subsection{Granular flows past a cuboid dam}

This section begins with comparing numerical and experimental results with respect to flows of dry Vestolen material past a cuboid dam and flows of yellow sand past a forward-facing tetrahedral wedge. The computation domain in numerical simulation follows the geometry of the chute, and it is discretized into 300 grids down-slope and 100 grids cross-slope, corresponding to the mesh resolution $\Delta x=5.67 \mathrm{~mm}$ and $\Delta y=11 \mathrm{~mm}$. Numerical tests demonstrate that the mesh of this resolution assures convergence of the numerical solution. A cuboid dam of height $80 \mathrm{~mm}$, length $160 \mathrm{~mm}$ and thickness $10 \mathrm{~mm}$ is vertically placed at the downslope position $x=650 \mathrm{~mm}$ from the top edge of the plane and in the middle of the inclined plane. Such an obstacle represents a blunt one commonly used in the field. It is necessary to note that this case looks very similar to that of Gray et al. [2003], however they are essentially different. A uniform incoming granular flow past a sharp and a blunt obstacle was investigated in Gray et al. [2003], in which runout dynamics were not investigated.

\section{(i) Dry granular flow}

Figs. 5 and 6 demonstrate the experimental images and surface velocity field measured by PIV technique, and the corresponding numerical predictions, respectively. Numerical results agree with experiment measurement pretty well. At the instance $(t=0.397 \mathrm{~s})$ when the grains have already impinged the obstacle, the flow increases in thickness. Both numerical and experimental results identify two oblique shock waves in the two sides of the obstacle and a third shock wave propagating in the upstream region of the obstacle. The third shock wave is very similar to that developed when the granular pile impinges on a rigid wall (see Pudasaini et al. [2007]). As the third shock propagates upstream, grains that passed by the shock wave will be deposited to develop a dead zone in front of the obstacle.

As grains continue to move down-slope, the avalanche front has reached the horizontal runout region at $t=0.663 \mathrm{~s}$ and is decelerated. Both numerical and theoretical results demonstrate that the maximum velocity occurs on the transition part and slightly upstream. At $t=0.93 \mathrm{~s}$, it is demonstrated that some grains deposit on the horizontal runout plane due to the absence of the driven force and two knolls develop at the two sides of the central line of the chute. At $t=1.197 \mathrm{~s}$, the two knolls merge together as the experimental result shows, which is also captured by numerical simulation. A grain-free region (i.e. vacuum) encircled by the mass is 
clearly shown. From practical point of view, it is very important to accurately predict such a vacuum. Numerical prediction and the experimental measurement agree well for the shape and size of the vacuum. The shape of the vacuum, observed here, distinguishes from that observed in Gray et al. [2003]. In Gray et al. [2003], the grain-free region is not closed due to the fact that the granular mass did not deposit in their configuration. At $t=1.464 \mathrm{~s}$, both numerical and experimental results predict that the flow becomes narrower, because more grains deposit on two knolls of the horizontal plane.

\section{(ii) Debris flow}

The initial height profile (32) is used for the mixture of grains and the liquid, and the same chute as that used in the numerical simulation of dry granular flows is used in this subsection. The parameters of the material and the values are listed in Table 1. In the following, we mainly report and interpret numerical results, because no available experimental data can be referred to. However, the granular behaviors, predicted by the current theory, show consistency with those observed in recent experiments with respect to granular flows immersed in water.

The numerical prediction of the flow field is presented in Fig. 7. Similar to dry granular flows (Fig. 6), the flow is diverted into two branches after the debris-flow front hits the obstacle and some mass deposit in front of the obstacle. As a loosely packed granular medium $\left(\phi_{s}<\phi_{c}\right)$ is released here, the bulk would deform towards a consolidated state, which causes the interstitial liquid to be expelled from voids. The result is that the pore fluid pressure is elevated from the original hydrostatic state immediately after the release of the mass (see Fig. 8). The elevated pore fluid pressure will mitigate the granular friction and as a result the grains can relatively easily spread. Consequently, the flow is extended wider in the cross-slope direction compared to dry granular flows (see comparison between Fig. 6 and Fig. 7).

The compressed behaviour begins to weaken later on and the excess pore fluid pressure therefore dissipates. As the mixture travels further downslope, grains demonstrate a dilatant behaviour, which causes that the ambient fluid is sucked into the voids between the grains. Consequently, a negative excess pore fluid pressure $\left(\lambda \leq \widetilde{\rho}_{f} / \bar{\rho} \leq 0.408\right)$ appears especially in the margin of the flow (see the results at $t=0.397 \mathrm{~s}$ in Fig. 9). This is a well-observed behavior in the experiments of granular flows immersed in water due to the pore fluid pressure feedback (Pailha and Pouliquen [2009], Rondon et al. [2011] and Wang et al. [2017]).

Additionally, as the grain-liquid mixture impinges on the obstacle, the mixture in the front will decelerate immediately (or even bounce back) and be compressed by the succeeding mass sliding downslope. As a result of this contracted behaviour, the interstitial liquid is squeezed and 
expelled, and the pore fluid pressure increases accordingly which implies that a positive pressure appears and an approximately full fluidisation arises in the front of the obstacle at $t=0.397 \mathrm{~s}$ and $0.663 \mathrm{~s}$. Subsequently, for $t>0.93 \mathrm{~s}$, the pore fluid pressure in front of the obstacle begins to dissipate and eventually the excess pore fluid pressure vanishes.

\subsection{Granular flows past a forward-facing tetrahedral wedge}

In this example, a forward-facing tetrahedral sharp wedge with height $200 \mathrm{~mm}$ and bottom-side length $160 \mathrm{~mm}$ is placed along the middle of the flow track at the downslope position $x=730$ $\mathrm{mm}$ (the apex of the wedge is at $x=720 \mathrm{~mm}$ ). Dry and fluid-saturated granular flows past this

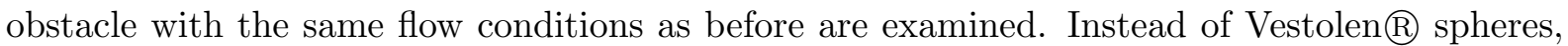
yellow sands is used as granular material.

\section{(i) Dry granular flow}

Figs. 10 and 11 demonstrate the experimental images and surface velocity fields measured by the PIV technique, and the corresponding numerical predictions. Comparing the front positions of dry sand flow between experimental and numerical results shows similar behaviour to that observed in the last subsection. The predicted front position and the velocity distribution overall agrees well with the experimental results. Additionally, it is found that the use of the tetrahedral wedge, different from the use of the cuboid dam, mainly diverts the flow direction rather than block the flow. Only a small dead zone is therefore observed in this case.

\section{(ii) Debris flow}

Further, a liquid saturated sand mixture is released from the cap. All the material parameters used in numerical simulation follow those listed in Table 1. Fig. 12 describes numerical predictions of the flow pattern and the velocity field. Similar to dry granular flows, the fluid-saturated flow is diverted after the mass hits the tetrahedral wedge and no dead zone is found. Comparison of results of the mixture and dry granular flows demonstrates again that the flow is extended wider in the cross-slope direction when the interstitial liquid is present, which is due to the coupling between the granular dilatancy and the pore fluid pressure.

Fig. 13 describes the spatial distribution of the dimensionless basal pore fluid pressure at several times. No granular liquefaction is found in front of the tetrahedral wedge. As analyzed above, a forward-facing tetrahedral wedge mainly diverts the flow rather than blocks the flow, and hence grains in the vicinity of the obstacle do not show apparent contacted behavior. Additionally, it is found again that grains in the margin of the flow are prone to dilate and 
therefore a depleted pore fluid pressure is found there.

\section{Conclusion and outlook}

The present paper numerically investigates dry granular and debris flows past an obstacle to gain insights into the physics behind them. To this end, we employ a continuum-mechanical fluid-granular mixture model taking the effects of the granular dilatancy and the pore fluid pressure into account. The obstacle is considered by a basal elevation from the reference plane. The resulting model equations are hyperbolic and hence they can be numerically solved by employing a shock-capturing scheme. To validate the model we investigate a large-scale experiment presented in Iverson et al. [2010]. The comparison of numerical results with experimental data demonstrates that the granular dilatancy that is linked with the development of excess pore fluid pressure plays a crucial role in the prediction of dynamic flow behaviors.

Furthermore, we investigate dry and wet granular past blunt and sharp obstacles. The experiments of dry granular flows past blunt and sharp obstacles have been performed and employed to scrutinize the current theory. The experiment of a dry granular flow past a cuboid dam shows that shock waves, dead zones and vacuum develop. These phenomena can also be reproduced by the present model. Additionally, numerical results also reveal that the use of a forward-facing tetrahedral wedge mainly diverts the flow, which is consistent with the experimental results.

The whole framework is further applied to study a fluid-saturated granular flow past the same obstacles as those used in dry granular flows. By comparing numerical results with those of dry granular flows, it is found that the pore fluid pressure feedback, i.e. the coupling of shear-induced granular dilatancy and the pore fluid pressure, plays a crucial role in different behaviors from those of dry granular flows. It implies that a debris flow is more spreading than a dry granular flow, which implicitly indicates that a debris flow is more destructive than a dry granular flow. Additionally, it is found that the presence of the obstacle, especially a blunt obstacle, has a significant influence on the elevation of the pore fluid pressure. The use of the blunt obstacle causes that grains in the upstream region of the blunt obstacle are prone to be compressed by succeeding mass sliding downslope, and hence the pore fluid is expelled and as a result the pore fluid pressure elevates. It in turn mitigates the granular friction. It is believed that insights gained here are helpful for understanding complex behaviors of geophysical flows and the current depth-integrated theory is promising to be applied to simulate geophysical flows, though numerical simulation of a debris flow past an obstacle is not validated by the experiment. In future work, it is necessary to design an experiment for a debris flow past an 
obstacle to scrutinize the current theory. Additionally, numerical simulation of a full-dimensional model (see Wang and Hutter [1999] and Heß et al. [2017] ) instead of depth-integrated model will be performed to remove limitations of the depth-integrated model.

\section{Acknowledgement}

The first author thank Marie Curie Individual fellowship programme of the European Union for funding support through grant No.792967. We are grateful to the reviewer Prof. K. Hutter from ETH Zurich for his linguistic corrections. The first author is thankful to Dr. Feng Feng from the Academy of Aerospace Aerodynamics for giving helpful suggestions to the manuscript.

\section{Data Availability Statement}

Some or all data, models, or code generated or used during the study are available from the corresponding author by request. Precisely, the experimental data of Fig. 3 and Fig. 8, and the computation code and data corresponding to Figs. 4-7, and 9-11 can be accessed from the corresponding author by request.

\section{Appendix A: The pore fluid pressure and the granular stress}

In shallow granular flows, it is commonly known that the use of the thin-layer assumption, i.e. "typical flow thickness much smaller than typical flow length", can transform the normal component of the momentum conservation equation into a force balance equation. It means that the normal components of the mixture momentum balance (2) and the fluid momentum balance (see eq. (29) in Meng and Wang [2018]) reduce to

$$
\begin{aligned}
& \frac{\partial}{\partial z}\left(T_{e(z z)}+p_{e}\right)=-\left(\rho-\widetilde{\rho}_{f}\right) g \cos \zeta, \\
& -\phi_{f} \frac{\partial p_{e}}{\partial z}=\frac{\mu_{f} \phi_{f}^{2}}{k}\left(w_{f}-w_{s}\right),
\end{aligned}
$$

respectively, where $p_{e}$ is the excess pore fluid pressure and it equals $p_{e}=p_{f}-\widetilde{\rho}_{f} g(\cos \zeta)(s-z)$.

Integrating (33) along the depth direction from any vertical position to the free surface yields

$$
T_{e(z z)}^{(z)}+p_{e}^{(z)}=\left(\rho-\widetilde{\rho}_{f}\right)(s-z) g \cos \zeta,
$$

where the traction-free condition (11), i.e. $T_{e(z z)}^{(s)}+p_{e}^{(s)}=0$, is used to simplify the integration. Similarly, integrating relation (34) along the depth direction leads to

$$
p_{e}^{(z)}=\frac{\mu_{f}}{k} \int_{z}^{s} \phi_{f}\left(w_{f}-w_{s}\right) d z
$$


where the difference of the normal velocity, $\left(w_{f}-w_{s}\right)$, remains to be formulated in order to obtain an analytical expression for $p_{e}^{(z)}$.

In standard mixture theory, the mass-balance equations of the solid and the fluid constituents are given by

$$
\frac{\partial \phi_{s}}{\partial t}+\nabla \cdot\left(\phi_{s} \boldsymbol{u}_{s}\right)=0, \quad \text { and } \quad \frac{\partial \phi_{f}}{\partial t}+\nabla \cdot\left(\phi_{f} \boldsymbol{u}_{f}\right)=0 \text {, }
$$

which implicitly assume that the solid and the fluid phases are incompressible such that intrinsic densities $\widetilde{\rho}_{s}$ and $\widetilde{\rho}_{f}$ are constant and have been already taken out from the mass-balance equations. Combination of the solid and the fluid mass-balance equations yields

$$
\nabla \cdot \boldsymbol{u}_{s}=\nabla \cdot \phi_{f}\left(\boldsymbol{u}_{s}-\boldsymbol{u}_{f}\right)
$$

Substituting (38) into dilatancy law (7) leads to

$$
\nabla \cdot \phi_{f}\left(\boldsymbol{u}_{s}-\boldsymbol{u}_{f}\right)=\dot{\gamma} \tan \psi
$$

Relation (39) can be expanded as follows

$$
\frac{\partial}{\partial z}\left(\phi_{f}\left(w_{s}-w_{f}\right)\right)=\dot{\gamma} \tan \psi
$$

as we postulate above that the fluid and the solid move with the same bed-aligned velocity.

Integrating (40) from the bed $b(x, y)$ to any vertical position can formulate the difference of the normal velocities. Substituting the velocity difference into (36) then leads to

$$
p_{e}^{(z)}=-\frac{\mu_{f}}{k} \int_{z}^{s}\left(\int_{b}^{z} \dot{\gamma} \tan \psi d z\right) d z,
$$

where the integrand $\dot{\gamma} \tan \psi$ can be approximated as $\dot{\gamma}_{b} \tan \psi_{b}$ (see eq.(34) in Meng and Wang [2018]), in which $\dot{\gamma}_{b}$ and $\tan \psi_{b}$ represent basal shear rate and tangent of the basal dilatancy angle, respectively. We follow Pailha et al. [2008] to postulate a parabolic velocity profile, so that the basal shear rate is written as $\dot{\gamma}_{b}=3 \sqrt{\bar{u}^{2}+\bar{v}^{2}} / h$ (Note that numerical results are not sensitive to the profile of linear shearing velocity or the parabolic velocity). By substituting $\dot{\gamma}_{b} \tan \psi_{b}$ into (41) we can derive the expression of the excess pore fluid pressure, which is written as

$$
p_{e}^{(z)}=-\frac{\mu_{f}}{2 k}\left(\dot{\gamma}_{b} \tan \psi_{b}\right)\left[h^{2}-(z-b)^{2}\right] .
$$

If this is substituted into (35), the solid effective stress is

$$
T_{e(z z)}^{(z)}=\left(\bar{\rho}-\widetilde{\rho}_{f}\right) g(\cos \zeta)(s-z)-p_{e}^{(z)} .
$$




\section{Appendix B: Depth-integration technique}

Integrating mass-balance equation (1) over the depth and applying the Leibnitz integration rule to interchange the orders of differentiation and integration, one can obtain the depth-averaged mass-balance equation,

$$
\frac{\partial(h \bar{\rho})}{\partial t}+\frac{\partial(h \overline{\rho u})}{\partial x}+\frac{\partial(h \overline{\rho v})}{\partial y}-\left(\rho \frac{\partial z}{\partial t}+\rho u \frac{\partial z}{\partial x}+\rho v \frac{\partial z}{\partial y}-\rho w\right)_{b}^{s}=0
$$

where $(f)_{b}^{s}$ represents the difference of the quantity $f$ evaluated on the top surface and on the base. The terms evaluated on the boundaries in (44) can be simplified by using the kinematic boundary conditions (10) and (12). This process yields

$$
\frac{\partial(h \bar{\rho})}{\partial t}+\frac{\partial(h \overline{\rho u})}{\partial x}+\frac{\partial(h \overline{\rho v})}{\partial y}=0 .
$$

Experimental results (Egashira et al. [2001]) show that volume fractions are almost uniformly distributed in the depth direction. As a result, the bulk density $\rho=\widetilde{\rho}_{s} \phi_{s}+\widetilde{\rho}_{f} \phi_{f}$ is independent on the $z$-coordinate. Equation (45) therefore reduces to

$$
\frac{\partial(h \bar{\rho})}{\partial t}+\frac{\partial(h \bar{\rho} \bar{u})}{\partial x}+\frac{\partial(h \bar{\rho} \bar{v})}{\partial y}=0 .
$$

Similarly, integrating the downslope and cross-slope components of the momentum equation (2) in the depth direction and then applying Leibnitz integration rule leads to the depthintegrated momentum equations. After performing the depth-integration technique, the lefthand side terms of the downslope component of the momentum equation (2) takes the form

$$
\begin{aligned}
\int_{b}^{s} L H S d z & =\frac{\partial(h \overline{\rho u})}{\partial t}+\frac{\partial\left(h \overline{\rho u^{2}}\right)}{\partial x}+\frac{\partial(h \overline{\rho u v})}{\partial y}-\left(\rho u \frac{\partial z}{\partial t}+\rho u^{2} \frac{\partial z}{\partial x}+\rho u v \frac{\partial z}{\partial y}-\rho u w\right)_{b}^{s} \\
& =\frac{\partial(h \bar{\rho} \bar{u})}{\partial t}+\frac{\partial(h \bar{\rho} \overline{u u})}{\partial x}+\frac{\partial(h \bar{\rho} \overline{u v})}{\partial y},
\end{aligned}
$$

where the kinematic boundary conditions (10) and (12) are used to simplify the terms on the boundaries. Additionally, terms $\overline{u^{2}}$ and $\overline{u v}$ need to be factorized. The constitutive relation (4) does not provide a link between shear stress and strain rate. In this case, we follow the approach, commonly used in the field of shallow granular flows, to introduce so-called Boussinesq factors $\chi_{\alpha}(\alpha=1,2,3)$. These terms are then factorized as follows,

$$
\overline{u^{2}}=\chi_{1} \bar{u} \bar{u}, \quad \overline{u v}=\chi_{2} \bar{u} \bar{v}, \quad \overline{v^{2}}=\chi_{3} \bar{v} \bar{v} .
$$

In general the Boussinesq factor $\chi_{\alpha}$ have distinct values, but should not differ too much from each another. We therefore choose $\chi_{\alpha}=\chi$ for all $\alpha$. Then, $\chi=1$ represents a plug flow, $\chi=4 / 3$ a linearly shearing profile with no-slip condition at the bottom, and $\chi=5 / 4$ a Bagnold velocity profile (Gray and Edwards [2014]). All the classical granular depth-integrated models, 
e.g. Savage and Hutter [1989], Pouliquen and Forterre [2002] and Gray and Edwards [2014], employ $\chi=1$, because non-unity values are incapable to identity grain-free regions (Hogg and Pritchard [2004]). Consequently, $\chi=1$ is adopted here.

The right-hand side terms of the down-slope component of the momentum equation (2), subject to depth integration, are expressible as

$$
\begin{aligned}
\int_{b}^{s} R H S d z= & -\frac{\partial}{\partial x}\left(h \bar{T}_{e(x x)}\right)-\frac{\partial}{\partial y}\left(h \bar{T}_{e(x y)}\right)+\frac{\partial}{\partial x}\left(h \bar{\phi}_{f} \bar{\tau}_{f(x x)}\right)+\frac{\partial}{\partial y}\left(h \bar{\phi}_{f} \bar{\tau}_{f(x y)}\right) \\
& +\left(T_{e(x x)} \frac{\partial z}{\partial x}+T_{e(x y)} \frac{\partial z}{\partial y}-T_{e(x z)}\right)_{b}^{s} \\
& -\left(-p_{f}+\tau_{f(x x)} \phi_{f} \frac{\partial z}{\partial x}+\tau_{f(x y)} \phi_{f} \frac{\partial z}{\partial y}-\phi_{f} \tau_{f(x z)}\right)_{b}^{s} \\
& -\frac{\partial}{\partial x}\left(h \bar{p}_{f}\right)+\bar{\rho} g h \sin \zeta
\end{aligned}
$$

where the terms on the free surface will vanish due to the constraint of traction-free condition (11), and the terms on the bottom become

$$
\begin{aligned}
& T_{e(x x)}^{(b)} \frac{\partial b}{\partial x}+T_{e(x y)}^{(b)} \frac{\partial b}{\partial y}-T_{e(x z)}^{(b)}=\frac{u_{s}^{(b)}}{\sqrt{\left(u_{s}^{(b)}\right)^{2}+\left(v_{s}^{(b)}\right)^{2}}}\left(\boldsymbol{n}^{(b)} \cdot \boldsymbol{T}_{e} \boldsymbol{n}^{(b)}\right) \mu_{s}+\left(\bar{\rho} g h \cos \zeta-p_{b e d}\right) \frac{\partial b}{\partial x} \\
& -p_{f}^{(b)}+\tau_{f(x x)}^{(b)} \phi_{f}^{(b)} \frac{\partial b}{\partial x}+\tau_{f(x y)}^{(b)} \phi_{f}^{(b)} \frac{\partial b}{\partial y}-\phi_{f}^{(b)} \tau_{f(x z)}^{(b)}=-k_{f}^{b} \phi_{f}^{(b)} u_{f}^{(b)}+p_{b e d} \frac{\partial b}{\partial x} .
\end{aligned}
$$

The bed solid normal stress $\boldsymbol{n}^{(b)} \cdot \boldsymbol{T}_{e} \boldsymbol{n}^{(b)}$ on the right-hand side of (50) can be derived through simplifying the normal component of the solid momentum balance equation. Usually, it is approximated as

$$
\boldsymbol{n}^{(b)} \cdot \boldsymbol{T}_{e} \boldsymbol{n}^{(b)}=\left(\bar{\rho}-\widetilde{\rho}_{f}\right) g h \cos \zeta-p_{e}^{(b)}+\left(\widetilde{\rho}_{s}-\widetilde{\rho}_{f}\right) \bar{\phi}_{s} \kappa h \bar{u}^{2},
$$

see eq. (45) in Meng and Wang [2016].

In the models of shallow granular flows, e.g. Gray et al. [1999], Meng and Wang [2016], etc., the stress gradient $\partial\left(h \bar{T}_{e(x y)}\right) / \partial y$, arising in (49), is usually ignored, since it is negligibly small . The integration $\partial\left(h \bar{T}_{e(x x)}\right) / \partial x$ is usually simplified by introducing an earth pressure coefficient $K_{x}$, which characterizes anisotropy of the solid effective stress. More precisely, Savage and Hutter [1989] follow conventional soil mechanics to postulate $T_{e(x x)}^{(z)}=K_{x} T_{e(z z)}^{(z)}$, where $K_{x}$ depends on the angle of granular internal friction and the angle of bed friction. Utilizing this relation and (43) to deduce depth-averaged stress $\bar{T}_{e(x x)}$, and then substituting $\bar{T}_{e(x x)}$ into the integration $\partial\left(h \bar{T}_{e(x x)}\right) / \partial x$ leads to

$$
\frac{\partial}{\partial x}\left(h \bar{T}_{e(x x)}\right)=\frac{\partial}{\partial x}\left[\frac{K_{x}}{2}\left(\bar{\rho}-\widetilde{\rho}_{f}\right) g(\cos \zeta) h^{2}-\frac{2 K_{x}}{3} h p_{e}^{(b)}\right] .
$$


In the following we prescribe $K_{x}=1$, since numerical results demonstrate a very small difference between the choices of anisotropic normal stresses and isotropic normal stresses (see Prochnow et al $[2000])$.

The integrations of the fluid shear stress in (49), i.e $\partial\left(h \bar{\phi}_{f} \bar{\tau}_{f(x x)}\right) / \partial x$ and $\partial\left(h \bar{\phi}_{f} \bar{\tau}_{f(x y)}\right) / \partial y$, can be deduced by following the above depth-integration procedure. However, we will omit these fluid shear-stress terms to simplify the model, since they are generally small quantities, which can be proved by conducting a dimensional analysis (see page 10 in Meng and Wang [2016]). Actually, they have been omitted in several depth-integrated models of debris flows, see Pitman and Le [2005] and Iverson and George [2014], except for discussing some subtle cases, e.g. predicting cutoff frequency of instability (Gray and Edwards [2014]), velocity profile across the cross-slope direction (Meng and Wang [2018]) etc.

By combination of the excess pore fluid pressure (42) and hydrostatic component $\widetilde{\rho}_{f} g(\cos \zeta)(s-$ $z$ ) one can formulate the pore fluid pressure $p_{f}$ and its depth-averaged form $\bar{p}_{f}$. Substitution of the result into the integration of $\partial\left(h \bar{p}_{f}\right) / \partial x$, arising in (49), leads to

$$
\frac{\partial}{\partial x}\left(h \bar{p}_{f}\right)=\frac{\partial}{\partial x}\left(\frac{1}{2} \widetilde{\rho}_{f} g(\cos \zeta) h^{2}+\frac{2}{3} h p_{e}^{(b)}\right) .
$$

Analogously by combining the relations (47) and (49) one can formulate the down-slope component of the depth-integrated momentum equations, in which relations (50)-(53) are employed to complement unknown terms. Similarly, one can repeat the above procedure to derive the cross-slope component of the depth-integrated momentum equations. The final depth-integrated momentum equations are shown in (17) and (18), where we prescribe that the bed velocity approximately equals the depth-averaged velocity.

\section{References}

Anderson, T.B., R. Jackson (1967), Fluid mechanical description of fluidized beds. Equations of motion, Ind. Eng. Chem. Fundam. 6, 527-539.

Albaba, A., S. Lambert, F. Nicot, and B. Chareyre (2015), Relation between microstructure and loading applied by a granular flow to a rigid wall using DEM modeling, Granular Matter, 17, 603-616.

Andreotti, B., Y. Forterre, O. Pouliquen. (2013), Granular media, between fluid and solid, Cambridge University Press, Cambridge.

Barker, F., D.G. Schaeffer, P. Bohorquez, J.M.N.T. Gray (2015), Well-posed and ill-posed behavior of the $\mu(I)$-rheology for granular flow, J. Fluid Mech., 779, 794-818.

Bouchut, F., E.D. Fernandez-Nieto, A. Mangeney, G. Narbona-Reina (2016), A two-phase two-layer model for fluidized granular flows with dilatancy effects, J. Fluid Mech., 801, 166-221. 
Canelli, L., A.M. Ferrero, M. Migliazza, and A. Segalini (2012), Debris flow risk mitigation by the means of rigid and flexible barriers-experimental tests and impact analysis, Nat. Hazards Earth Syst. Sci., 12, 1693-2012.

Choi, C.E., C.W.W. Ng, R.P.H. Law, D. Song, J.S.H. Kwan, and K.K.S. Ho (2014), Computational investigation of baffle configuration on impedance of channelized debris flows, Can. Geotech. J., 52, $182-197$.

Chu, T., G. Hill, D.M. McClung, R. Ngun, and R. Sherkat (1995), Experiments on granular flows to predict avalanche runup, Can. Geotech. J., 32, 285-295.

Chiou, M.C. (2006), Modelling dry granular avalanches past different obstructs: numerical simulations and laboratory analyses, Dissertation, Techanical University Darmstadt, Germany.

Chiou, M.C., Y. Wang, and K. Hutter (2005), Influence of obstacles on rapid granular flows, Acta Mech, 175, 105-122.

Cui, X., and J.M.N.T Gray (2013), Gravity-driven granular free-surface flow around a circular cylinder, J. Fluid Mech., 720, 314-337.

Denlinger, R.P., and R.M. Iverson (2001), Flow of variably fluidized granular masses across threedimensional terrain. 2. Numerical predictions and experimental tests, J. Geophys. Res., 106, 552.

Egashira, S., T. Itoh, and H. Takeuchi (2001), Transition mechanism of debris flows over rigid bed to over erodible bed, Phys. Chem. Earth B, 26, 169-174.

Faug, T., P. Lachamp, and M. Naaim (2002), Experimental investigation on steady granular flows interacting with an obstacle down an inclined channel: study of the dead zone upstream from the obstacle. Application to interaction between dense snow avalanches and defence structures, Phys. Fluids, 2, 187-191.

Forterre, Y., and O. Pouliquen (2008), Flows of dense granular media, Annu. Rev. Fluid Mech., 40, 1-24.

George, D.L. (2008), Augmented Riemann solvers for the shallow water equations over variable topography with steady states and inundation, J. Compu. Phys., 227(6), 3089-3113.

Guazzelli, E., O. Pouliquen (2018), Rheology of dense granular suspensions, J. Fluid Mech., 852 1-73.

Gray, J.M.N.T., M. Wieland, K. Hutter (1999), Gravity-driven free surface flow of granular avalanches over complex basal topography, Proc. R. Soc. Lond. A, 445 1841-1874.

Gray, J.M.N.T., Y.C. Tai, S.Noelle (2003), Shock waves, dead-zones and particle-free regions in rapid granular free surface flows, J. Fluid Mech., 491, 161-181.

Gray, J.M.N.T., and A.N. Edwards (2014), A depth-averaged $\mu(I)$-rheology for shallow granular freesurface flows, J. Fluid Mech., 755, 503-534.

George, D.L., and R.M. Iverson (2014), A depth-averaged debris-flow model that includes the effects of evolving dilatancy. II. Numerical predictions and experimental tests, Proc. R. Soc. Lond. A, 470, 2170.

Heß, J., Y. Wang, K. Hutter (2017), Thermodynamically consistent modeling of granular-fluid mixtures incorporating pore pressure evolution and hypoplastic behavior, Continuum Mech. Therm., 29, 311343. 
Hogg, A., D. Pritchard (2004), The effects of hydraulic resistance on dam-break and other shallow inertial flows, J. Fluid Mech., 501, 179-212.

Iverson, R.M., D.L. George (2014), A depth-averaged debris-flow model that includes the effects of evolving dilatancy. I. physical basis, Proc. R. Soc. Lond. A, 470, 20130819.

Iverson, R.M. (1997), The physics of debris flows, Rev. Geophys., 35, 245-296

Iverson, R.M. (2003), The debris-flow rheology myth. In Debris-flow hazards mitigation: mechanics, prediction, and assessment, vol. 1 (eds D Richenmann, CL Chen), pp.303-314. Rotterdam, The Netherlands: Millpress.

Iverson, R.M., M. Logan, R.G. LaHusen, M.Berti (2010), The perfect debris flow? aggregated results from 28 large-scale experiments, J. Geophy. Res., 115, F03005.

Iverson, R.M., R.P. Denlinger (2001), Flow of variably fluidized granular masses across three-dimensional terrain: 1. Coulomb mixture theory, J. Geophys. Res., 106 (B1), 537-552.

Iverson, R.M., M.E. Reid, N.R. Iverson, R.G. LaHusen, M. Logan, J.E. Mann, D.L. Brien (2000), Acute sensitivity of landslide rates to initial soil porosity, Sci., 290, 513-516.

Jop, P., Y. Forterre, and O. Pouliquen (2006), A constitutive relation for dense granular flows, Nature, 44, 727-730.

Kattel, P., J. Kafle, J.T. Fischer, M. Mergili, B.M. Tuladhar, and S.P. Pudasaini (2018), Interaction of two-phase debris flow with obstacles, Eng. Geol., 242, 197-217.

Leonardi, A., F.K. Wittel, M. Mendoza, R. Vetter, and H.J. Herrmann (2016), "Particle-Fluid-Structure Interaction for Debris Flow Impact on Flexible Barriers", Comput.-Aided Civ. Infrastruct Eng., 31, 323-333.

Kuo, C.Y., L.T. Sheng, S.Y. Chiu, Y.Z. Yang, Y.C. Tai, and S.S. Hsiau (2015), Measurement and discrete element simulation of a fixed-obstacle disturbed rapid granular chute flow, Phys. Fluids, 27, 013305.

Meng, X., Y. Wang (2018), Modeling dynamic flows of grain-fluid mixtures by coupling the mixture theory with a dilatancy law, Acta Mech., 229, 2521-2538.

Meng, X., Y. Wang (2016), Modelling and numerical simulation of two-phase debris flows, Acta Geotech., $11,1027-1045$.

Meng, X., Y. Wang, C. Wang, J-T. Fischer (2017), Modeling of unsaturated granular flows by a two-layer approach, Acta Geotech., 12, 677-701.

Meng, X., Y. Wang, F. Feng, C. Wang, and Y. Zhou, Granular flows in a rotating drum and on an inclined plane: Analytical and numerical solutions, Phys. Fluids, 30, 106605 (2018).

Moriguchi, S., R.I. Borja, A. Yashima, and K. Sawada (2009), Estimating the impact force generated by granular flow on a rigid obstruction, Acta Geotech., 4, 57-71.

Nessyahu, H., E. Tadmor (1990), Non-oscillatory central differencing for hyperbolic conservation laws, J. Comput. Phys., 87, 408-463.

Pailha, M.,M. Nicolas, O. Pouliquen (2008), Initiation of underwater granular avalanches: influence of the initial volume fraction, Phys. Fluids, 20, 111701. 
Pailha, M., O. Pouliquen (2009), A two-phase flow description of the initiation of underwater granular avalanches, J. Fluid Mech., 633, 115-135.

Pitman, E.B., and L. Le (2005), A two-fluid model for avalanche and debris flows, Phil. Trans. R. Soc. A, 363, 1573-1601.

Prochnow, M., F. Chevoir, M. Albertelli (2000), Dense granular flows down a rough inclined plane, In Proceedings of XIII International Congress on Rheology, Cambridge, UK.

Pouliquen, O. (1999), On the shape of granular fronts down rough inclined planes, Phys. Fluids, 11, 1956-1958

Pouliquen, O., and Y. Forterre (2002), Friction law for dense granular flows: application to the motion of a mass down a rough inclined plane, J. Fluid Mech., 25, 133-151.

Pudasaini, S.P., S.S Hsiau, Y. Wang, and K. Hutter (2005), Velocity measurements in dry granular avalanches using Particle Image Velocimetry-Technique and comparison with theoretical predictions, Physc. Fluids, 17, 093301.

Pudasaini, S.P., K. Hutter, Y. Wang, and R. Katzenbach (2007), Rapid flow of dry granular materials down inclined chutes impinging on rigid walls, Physc. Fluids, 19, 053302.

Pudasaini, S.P. (2012), A general two-phase debris flow model, J Geophys. Res, 117, F3.

Rondon, L., O. Pouliquen, and P. Aussillous (2011), Granular collapse in a fluid: role of the initial volume fraction, Phys. Fluids, 23, 073301.

Roux, S., and F. Radjai (1998), Texture-dependent rigid plastic behavior. In Proceedings: Physics of Dry Granular Media, September 1997, Cargése, France, pp. 305-311 (eds. H. J. Herrmann et al.) Kluwer.

Song, D., C.W.W. Ng, C.E Choi, G.G.D. Zhou, J.S.H. Kwan, and R.C.H. Koo (2017), Influence of debris flow solid fraction on rigid barrier impact, Can. Geotech. J., 54, 1421-1434.

Savage, S. B., and K. Hutter (1989), The motion of a finite mass of granular material down a rough incline, J. Fluid. Mech., 199, 177-215.

Teufelsbauer, H., Y. Wang, M.C. Chiou, and W. Wu (2009), "Flow-obstacle interactions in rapid granular avalanches: DEM simulations and comparison with experiments", Granul. Matter, 11, 209-220.

Teufelsbauer, H., Y. Wang, S.P. Pudasaini, R.I. Borja, and W. Wu (2011), "DEM simulation of impact force exerted by granular flow on rigid structures", Acta Geotech., 6, 119-133.

Tai, Y.C., S. Noelle, J.M.N.T. Gray, K. Hutter (2001), Shock-capturing and front-tracking methods for granular avalanches, J. Compu. Phys., 175, 269-301.

Tai, Y.C., J. Heß, and Y. Wang (2019), Two-phase debris flow over rugged topography: Modelling and numerical simulation, J. Geophys. Res., 124, 305-333.

Truesdell, C. (1992), A first course in rational continuum mechanics, Academic Press.

Wang, Y., and K. Hutter (1999), A constitutive theory of fluid-saturated granular materials and its application in gravitational flows, Rheol Acta., 38, 214-223.

Wang, Y., K. Hutter, and S. P. Pudasaini (2004), The Savage-Hutter theory: A system of partial differential equations for avalanche flows of snow, debris, and mud, J. App. Math. Mech., 84(8), 507-527. 
769 Wang, C., and Y. Wang, C. Peng, and X. Meng (2017), Dilatancy and compaction effects on the sub770 merged granular column collapse, Phys. Fluids, 29, 103307. 


\section{${ }_{771}$ List of Tables}

$772 \quad 1$ Material properties used in the numerical computation . . . . . . . . . 27

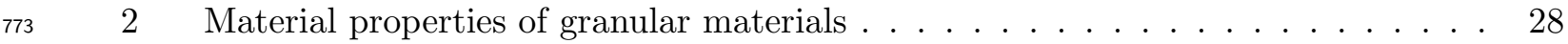


Table 1: Material properties used in the numerical computation

\begin{tabular}{lll}
\hline \hline Property & Experimental values & Model values \\
\hline Fluid density, $\widetilde{\rho}_{f}\left(\mathrm{~kg} / \mathrm{m}^{3}\right)$ & 1100 & 1100 \\
Solid density, $\widetilde{\rho}_{s}\left(\mathrm{~kg} / \mathrm{m}^{3}\right)$ & 2700 & 2700 \\
Initial solid volume fraction, $\phi_{s}$ & $0.61 \pm 0.04$ & 0.61 \\
Critical solid volume fraction, $\phi_{c}$ & 0.64 & 0.64 \\
Initial basal pore pressure, $p_{b e d}(\mathrm{~Pa})$ & hydrostatic & hydrostatic \\
Initial hydraulic permeability, $k\left(\mathrm{~m}^{2}\right)$ & $4 \times\left(10^{-12} \sim 10^{-11}\right)$ & $1 \times 10^{-8}$ \\
Pore fluid viscosity, $\mu_{f}(\mathrm{~Pa} \cdot \mathrm{s})$ & $0.001 \sim 0.05$ & 0.01 \\
Basal frictional coefficient, $k_{f}^{b}\left(\mathrm{~N} \cdot \mathrm{s} / \mathrm{m}^{3}\right)$ & - & 50 \\
\hline
\end{tabular}


Table 2: Material properties of granular materials

\begin{tabular}{l|llll}
\hline \hline & diameter $\mathrm{d}(\mathrm{mm})$ & $\rho\left(\mathrm{kg} / \mathrm{m}^{3}\right)$ & Mass $(\mathrm{kg})$ & basal angle $\delta$ \\
\hline Vestolen & 4 & 639 & 1.41 & $24^{\circ}$ \\
Yellow sand & Fine & 1661 & 3.75 & $27^{\circ}$ \\
\hline
\end{tabular}




\section{List of Figures}

1 The curvilinear coordinate system oxyz. The downslope coordinate $x$ is curvilinear, while the cross-slope coordinate $y$ is rectilinear. A topographical elevation $b(x, y)$ is imposed onto the reference plane. This figure is a reproduction of Figure 2 in Meng and Wang [2017] . . . . . . . . . . . . . . . . . . 31

2 Initial sand-gravel-mud mixture placed behind a vertical gate which is at the origin of the present coordinate system . . . . . . . . . . . . . . . . . 32

3 Time series of the depth profile at different locations downslope. The shaded areas represent experimental data, while the solid lines denote the numerical results from the present model, and the dashed lines represent the results without the granular dilatancy. . . . . . . . . . . . . . . . . . . . 33

4 Experiment set-up in the laboratory . . . . . . . . . . . . . . . . 34

5 Photography from the experiment of dry granular flow past a cuboid dam (panel a) and the velocity field of PIV measurement (panel b) at several times. The color in panel (b) indicates the value of the surface velocity. These experimental results are well documented in the $\mathrm{PhD}$ thesis of Chiou [2006]. All experiments have been performed in the laboratory at the Technical University of Darmstadt

$6 \quad$ Numerical prediction of the velocity field of dry granular flow past a cuboid dam. The results shown here correspond to those truncated from $x>0.45 \mathrm{~m}$. The color represents the distribution of the resultant depth-averaged velocity $\sqrt{\bar{u}^{2}+\bar{v}^{2}}$. .

$7 \quad$ Numerical prediction of velocity field of the liquid-grain mixture flow past a cuboid dam. The results shown here correspond to those truncated from $x>0.45 \mathrm{~m}$. The color represents the distribution of the resultant depth-averaged velocity $\sqrt{\bar{u}^{2}+\bar{v}^{2}} \cdot 37$

8 Short-term evolution of the pore fluid pressure. The color indicates the distribution of the dimensionless basal pore pressure $\lambda=p_{\text {bed }} /(\bar{\rho} g h \cos \zeta)$, in which $\lambda=0$ represents a depleted pore fluid pressure and $\lambda=1$ denotes a full granular

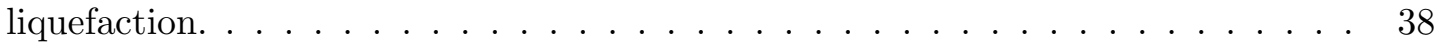

9 Three dimensional geometries of liquid-grain mixture flows at times $t=0.13 \mathrm{~s}$, $0.397 \mathrm{~s}, 0.663 \mathrm{~s}, 0.93 \mathrm{~s}, 1.197 \mathrm{~s}$, and $1.464 \mathrm{~s}$, consecutively. The color indicates the distribution of the dimensionless basal pore pressure $\lambda=p_{\text {bed }} /(\bar{\rho} g h \cos \zeta)$. . . . 
10 Photographs from the experiment (panel a) and the velocity fields of PIV measurement (panel b) at several times. The color in panel (b) indicates the value of the surface velocity. These experimental results are well documented in the $\mathrm{PhD}$ thesis of Chiou [2006]. All experiments have been performed in the laboratory at the Technical University of Darmstadt . . . . . . . . . . . . . . . . . . . . . . 40

11 Numerical prediction of velocity field of dry granular flow. The velocity field is demonstrated by the color. . . . . . . . . . . . . . . . . . . . . . . . . 41

12 Numerical prediction of the liquid-grain depth profile. The velocity field is demonstrated by the color. . . . . . . . . . . . . . . . . . . . . . . . 42

13 Three dimensional geometries of liquid-grain mixture flows at times $t=0.1 \mathrm{~s}$, $0.367 \mathrm{~s}$, and $0.634 \mathrm{~s}$, consecutively. The color indicates the distribution of the dimensionless basal pore fluid pressure $\lambda=p_{\text {bed }} /(\rho g h \cos \zeta) \ldots \ldots \ldots$ 


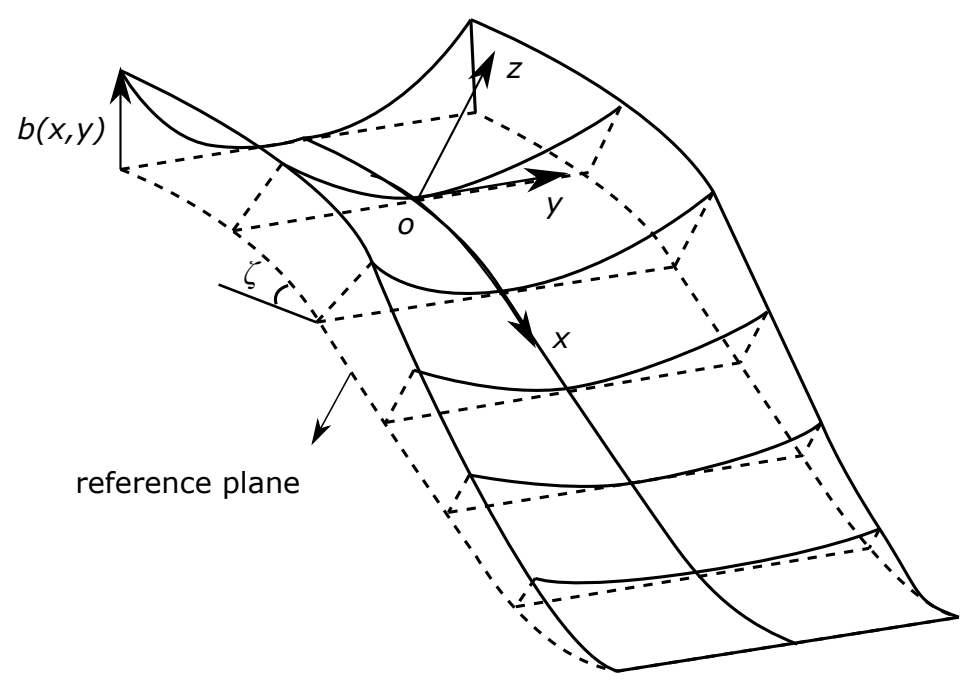

Figure 1: The curvilinear coordinate system oxyz. The downslope coordinate $x$ is curvilinear, while the cross-slope coordinate $y$ is rectilinear. A topographical elevation $b(x, y)$ is imposed onto the reference plane. This figure is a reproduction of Figure 2 in Meng and Wang [2017] 


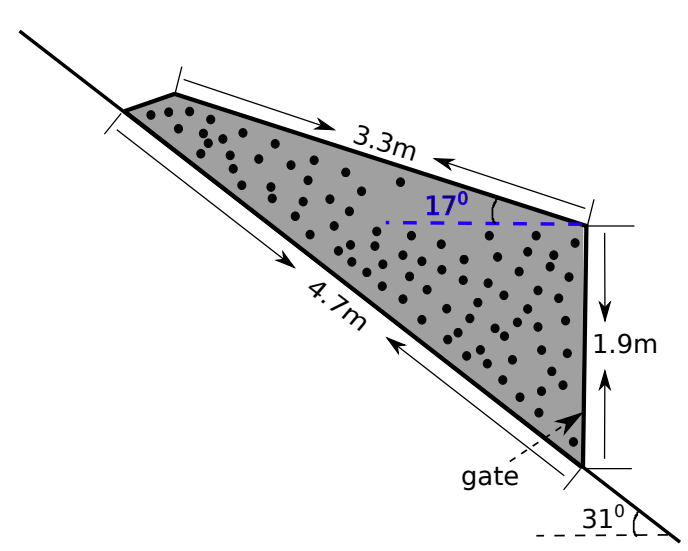

Figure 2: Initial sand-gravel-mud mixture placed behind a vertical gate which is at the origin of the present coordinate system 


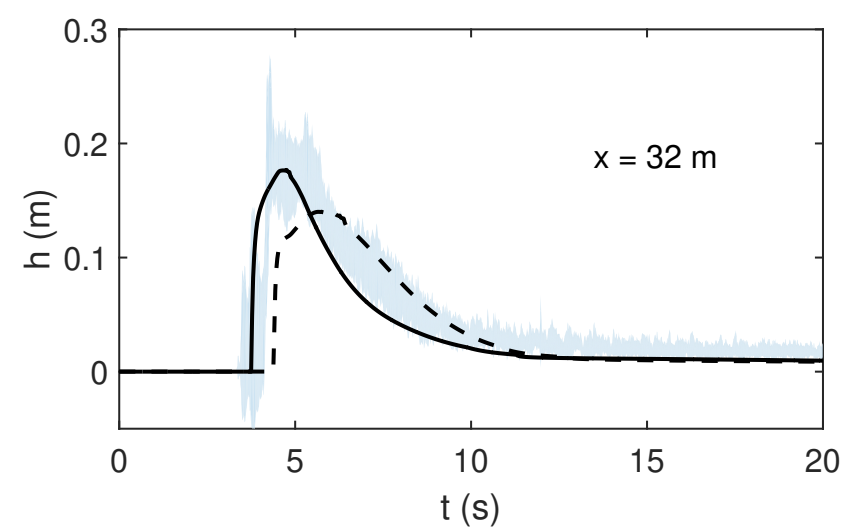

(a) Depth profile at $\mathrm{x}=32 \mathrm{~m}$

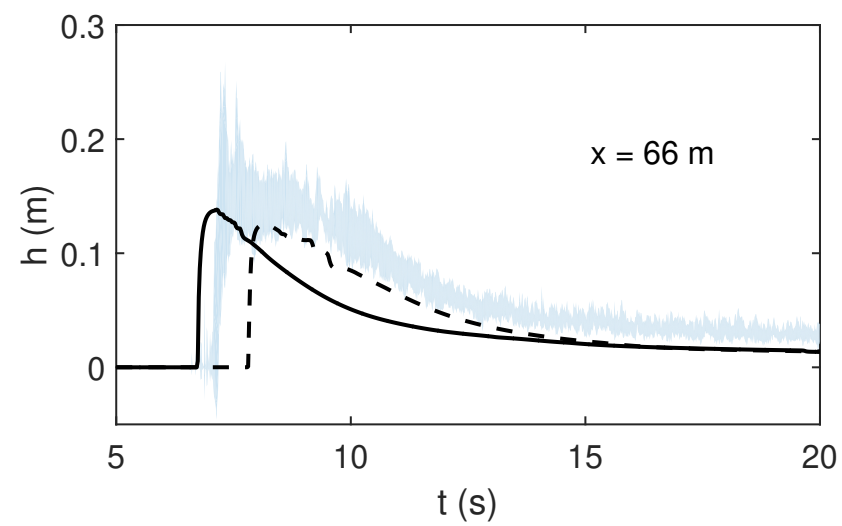

(b) Depth profile at $\mathrm{x}=66 \mathrm{~m}$

Figure 3: Time series of the depth profile at different locations downslope. The shaded areas represent experimental data, while the solid lines denote the numerical results from the present model, and the dashed lines represent the results without the granular dilatancy. 
(a) Experiment chute
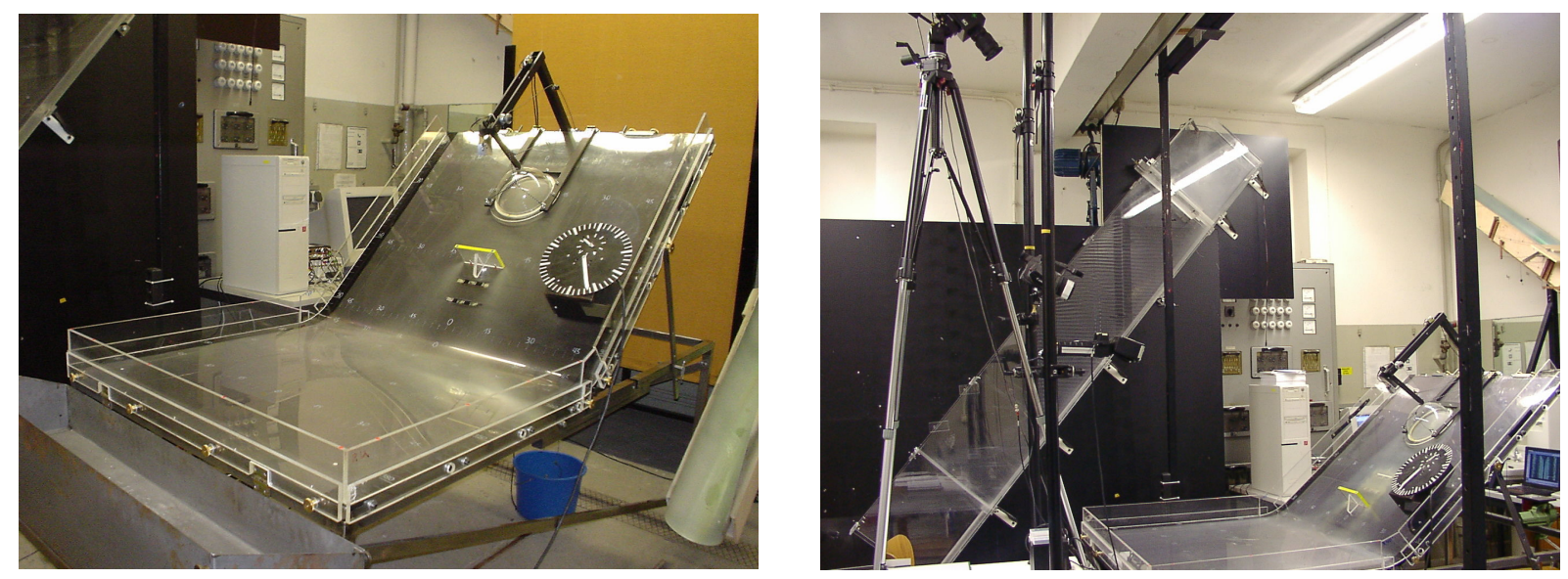

Figure 4: Experiment set-up in the laboratory 
(a) Photography from the experiment
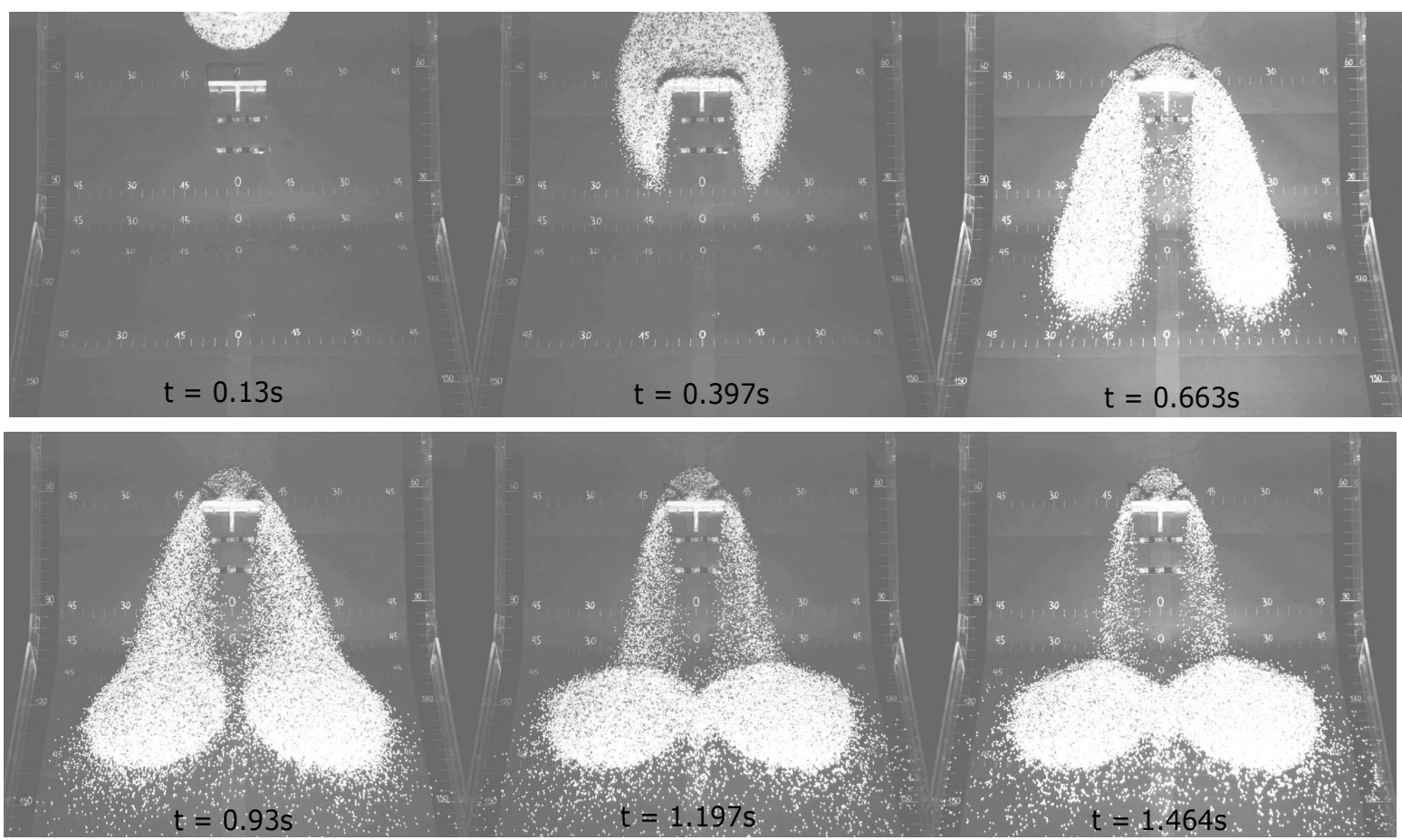

(b) Velocity field of PIV measurement
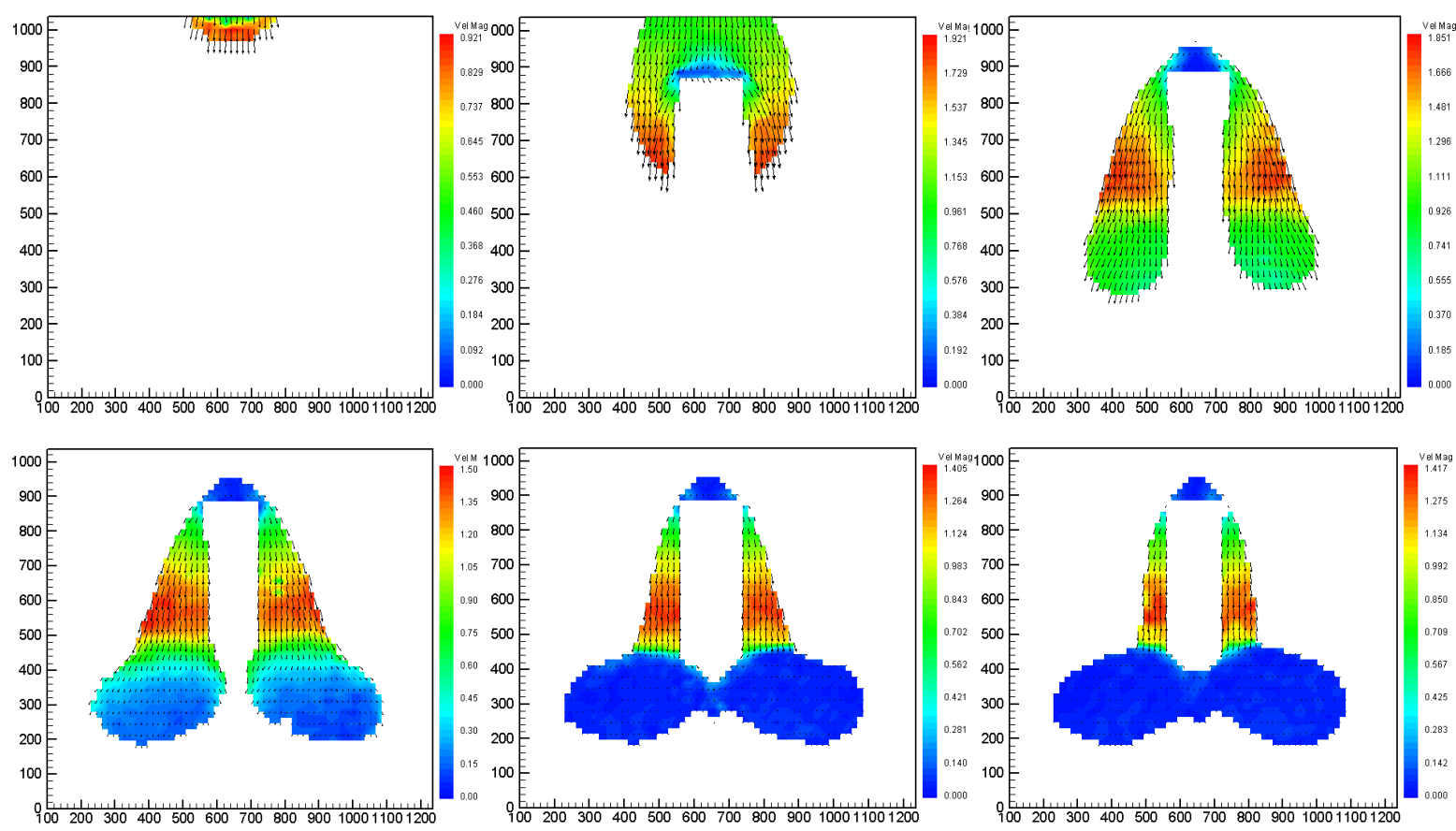

Figure 5: Photography from the experiment of dry granular flow past a cuboid dam (panel a) and the velocity field of PIV measurement (panel b) at several times. The color in panel (b) indicates the value of the surface velocity. These experimental results are well documented in the PhD thesis of Chiou [2006]. All experiments have been performed in the laboratory at the Technical University of Darmstadt 

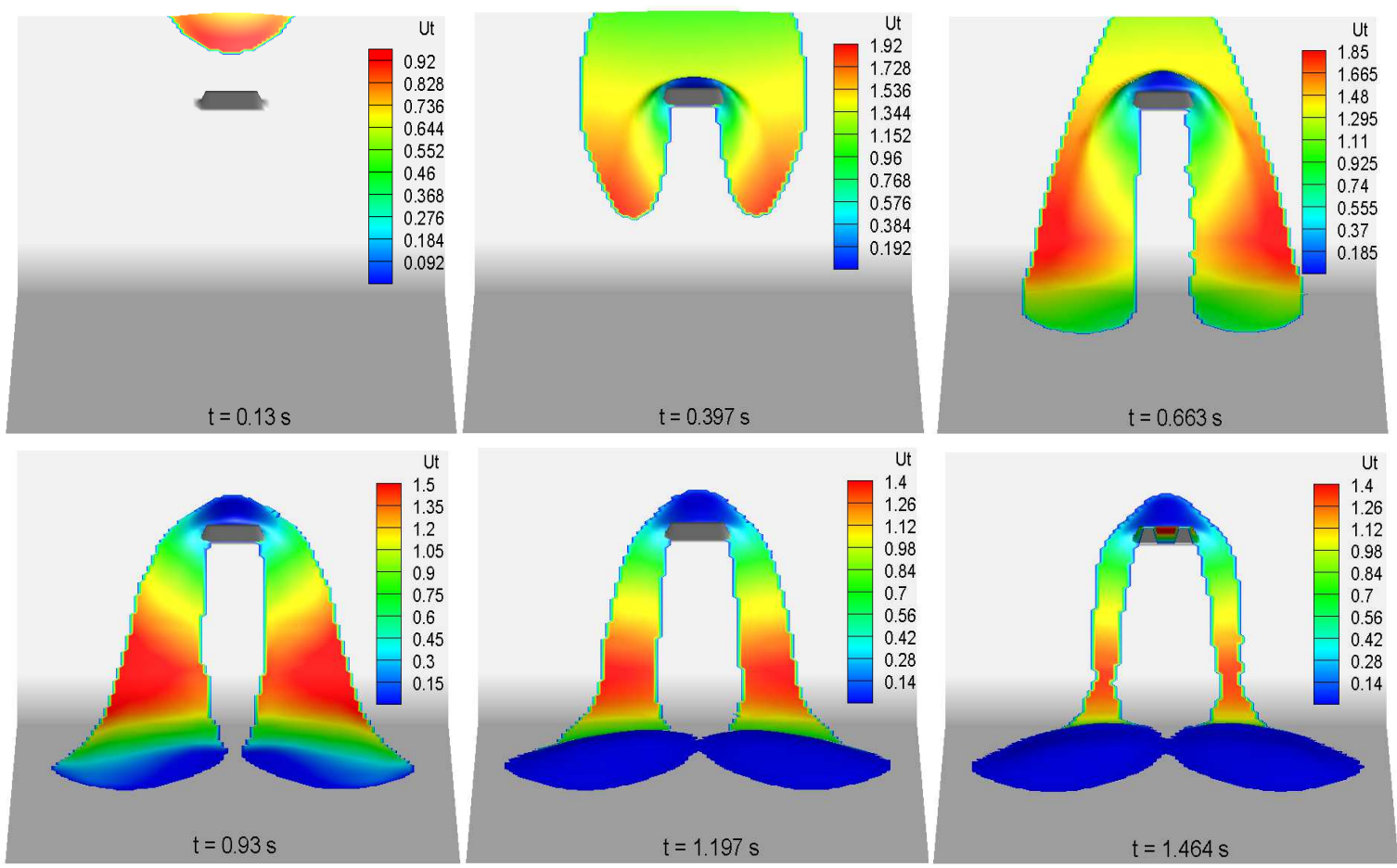

Figure 6: Numerical prediction of the velocity field of dry granular flow past a cuboid dam. The results shown here correspond to those truncated from $x>0.45 \mathrm{~m}$. The color represents the distribution of the resultant depth-averaged velocity $\sqrt{\bar{u}^{2}+\bar{v}^{2}}$. 


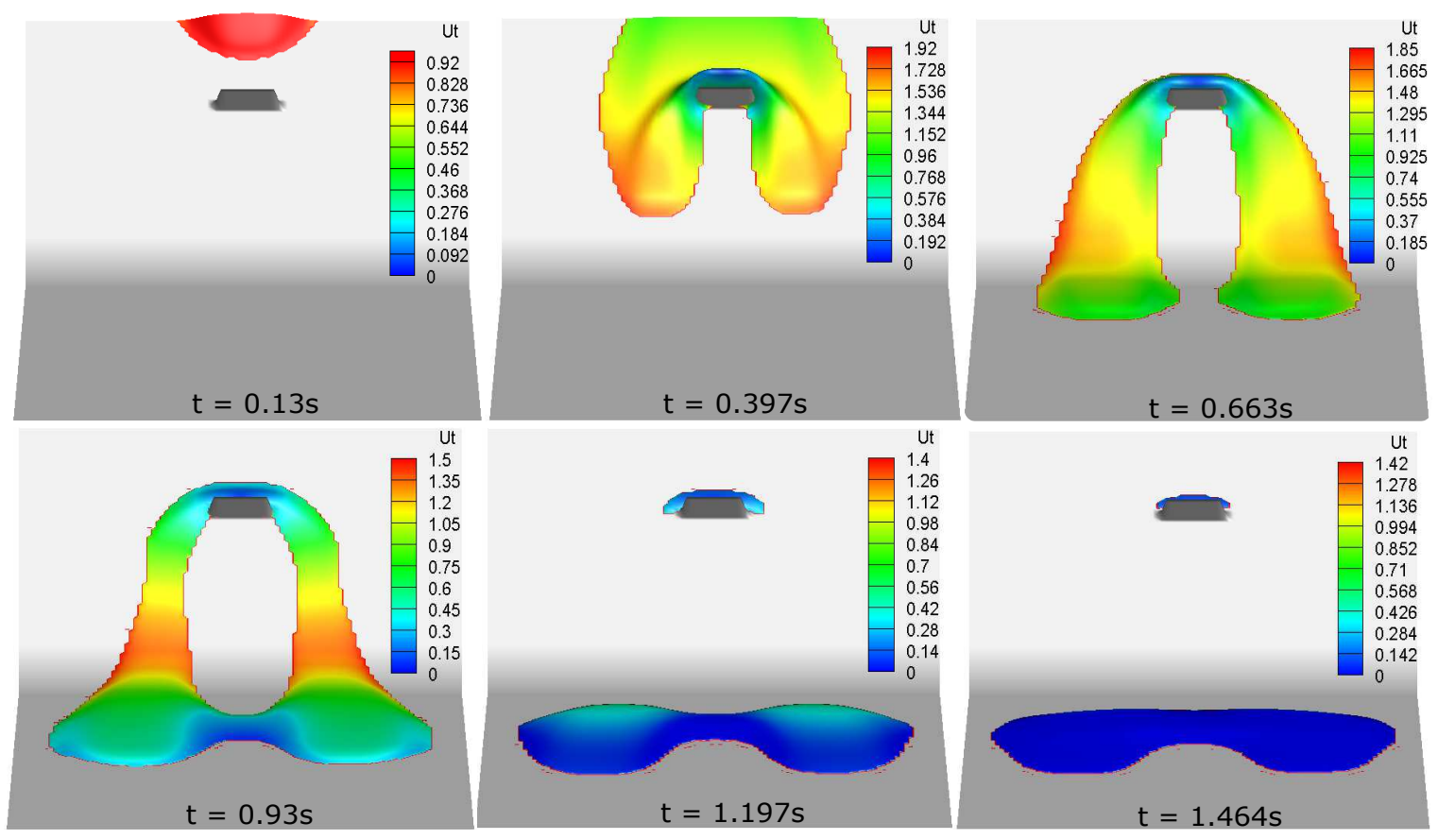

Figure 7: Numerical prediction of velocity field of the liquid-grain mixture flow past a cuboid dam. The results shown here correspond to those truncated from $x>0.45 \mathrm{~m}$. The color represents the distribution of the resultant depth-averaged velocity $\sqrt{\bar{u}^{2}+\bar{v}^{2}}$. 


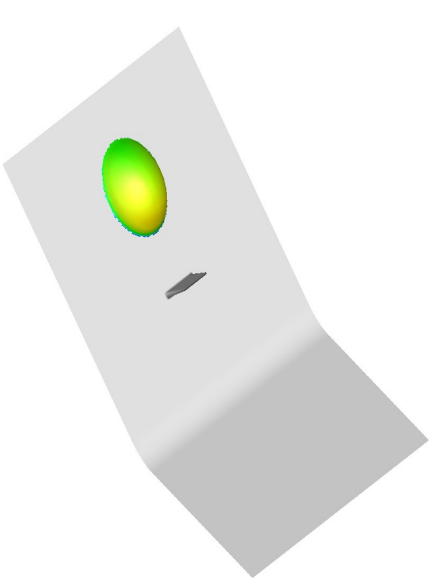

(a) $\mathrm{t}=0.01 \mathrm{~s}$

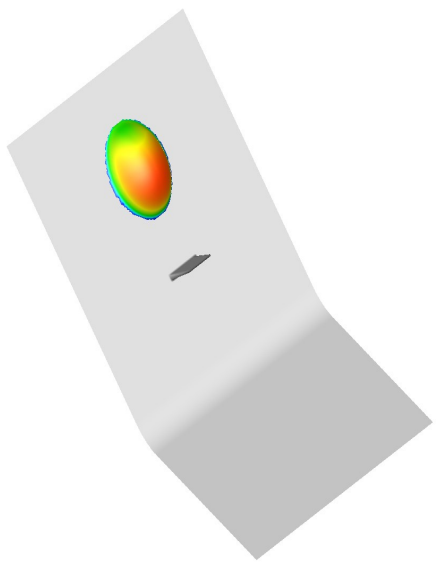

(b) $\mathrm{t}=0.03 \mathrm{~s}$

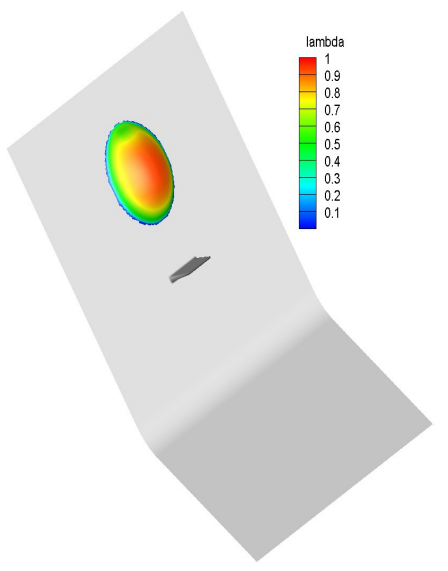

(c) $\mathrm{t}=0.06 \mathrm{~s}$

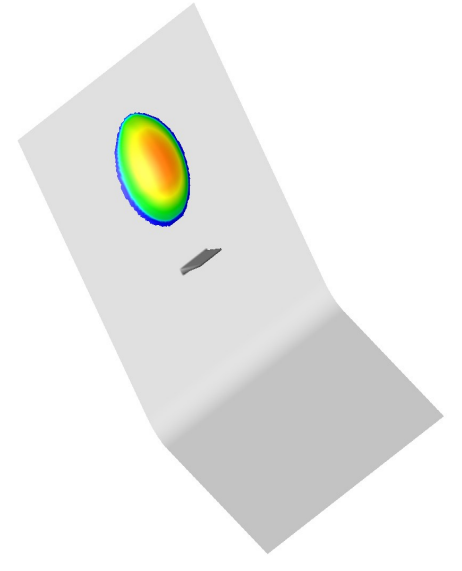

(d) $\mathrm{t}=0.09 \mathrm{~s}$

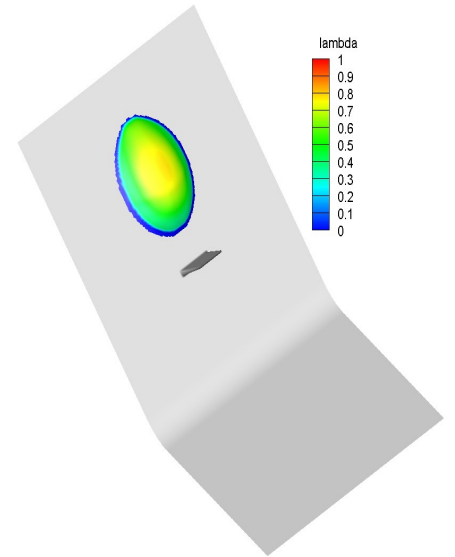

(e) $\mathrm{t}=0.12 \mathrm{~s}$

Figure 8: Short-term evolution of the pore fluid pressure. The color indicates the distribution of the dimensionless basal pore pressure $\lambda=p_{\text {bed }} /(\bar{\rho} g h \cos \zeta)$, in which $\lambda=0$ represents a depleted pore fluid pressure and $\lambda=1$ denotes a full granular liquefaction. 


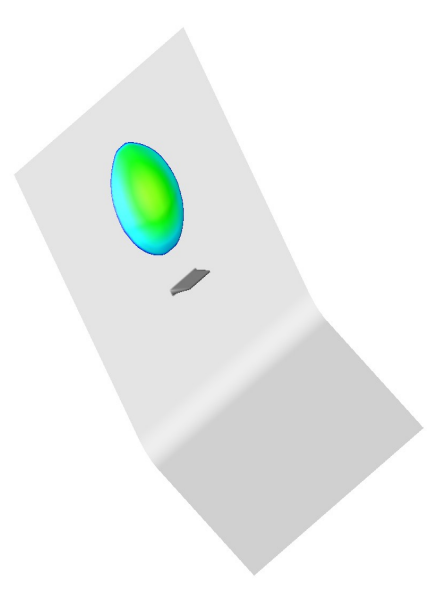

(a) $\mathrm{t}=0.13 \mathrm{~s}$

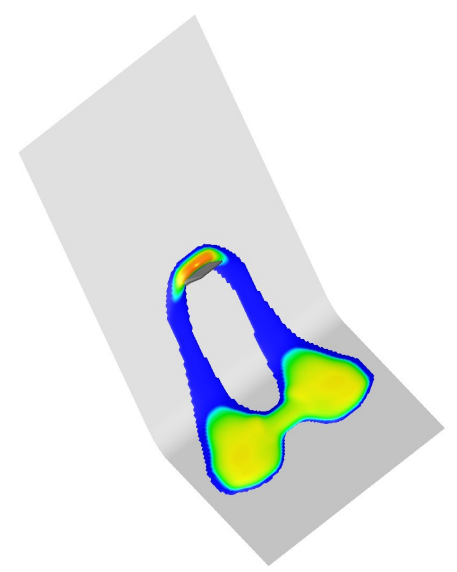

(d) $\mathrm{t}=0.93 \mathrm{~s}$

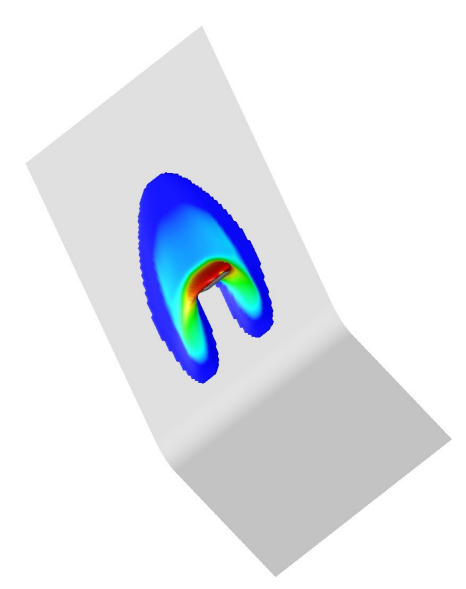

(b) $\mathrm{t}=0.397 \mathrm{~s}$

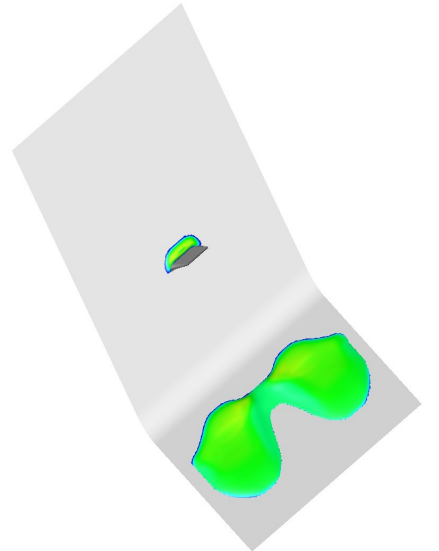

(e) $\mathrm{t}=1.197 \mathrm{~s}$

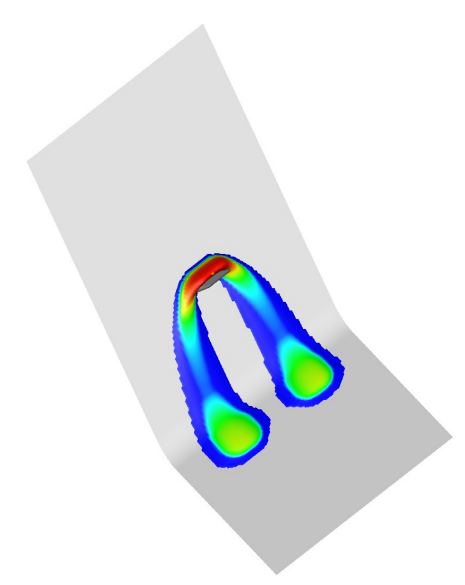

(c) $\mathrm{t}=0.663 \mathrm{~s}$

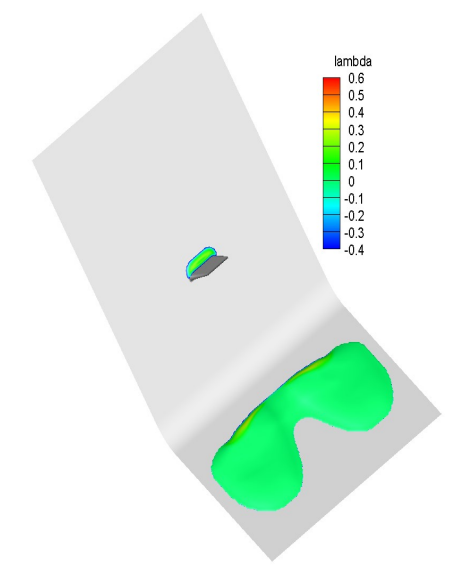

(f) $\mathrm{t}=1.464 \mathrm{~s}$

Figure 9: Three dimensional geometries of liquid-grain mixture flows at times $t=0.13 \mathrm{~s}, 0.397 \mathrm{~s}$, $0.663 \mathrm{~s}, 0.93 \mathrm{~s}, 1.197 \mathrm{~s}$, and $1.464 \mathrm{~s}$, consecutively. The color indicates the distribution of the dimensionless basal pore pressure $\lambda=p_{\text {bed }} /(\bar{\rho} g h \cos \zeta)$. 
(a) Photograph from the experiment
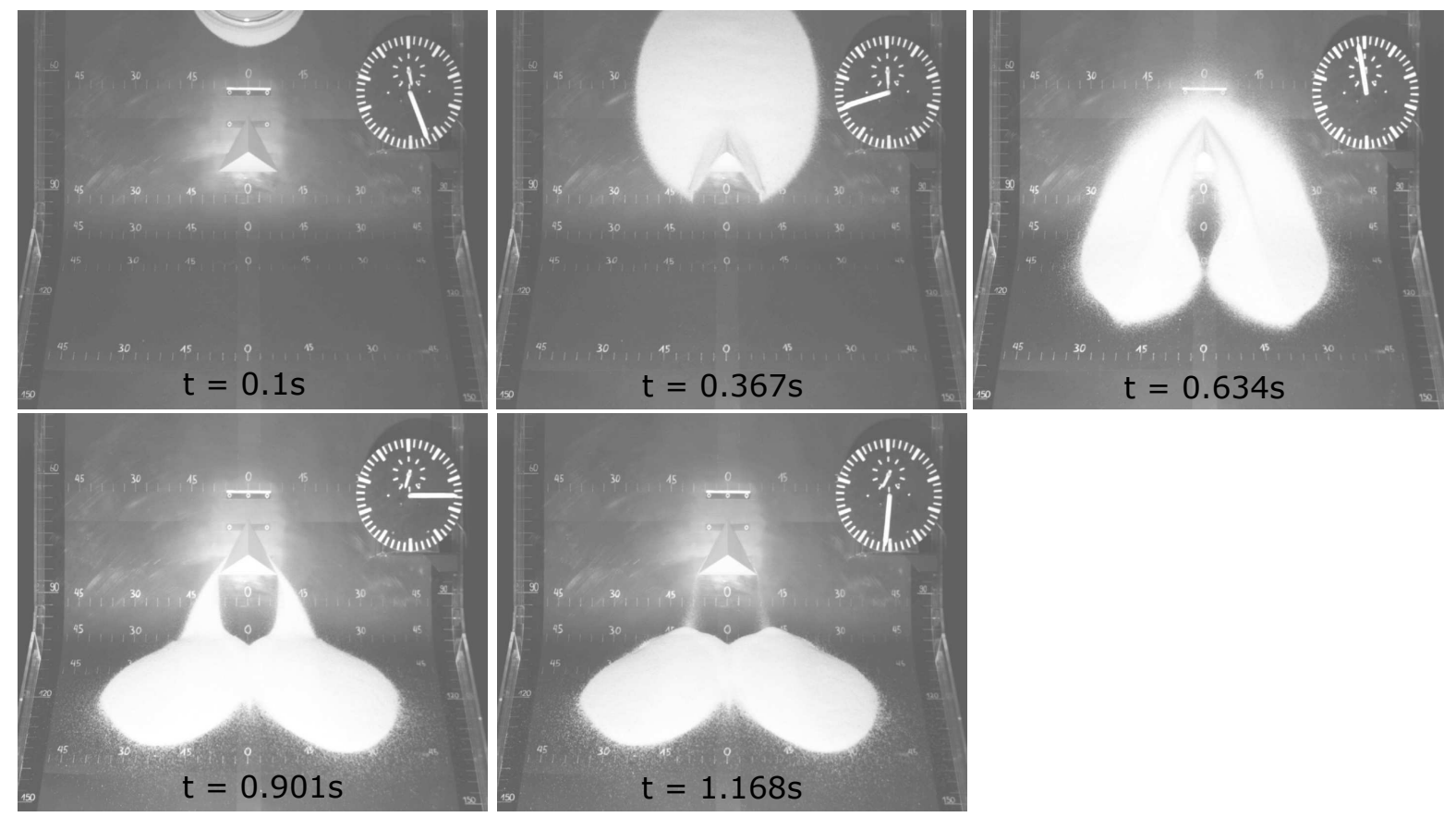

(b) Dry granular velocity field of PIV measurement

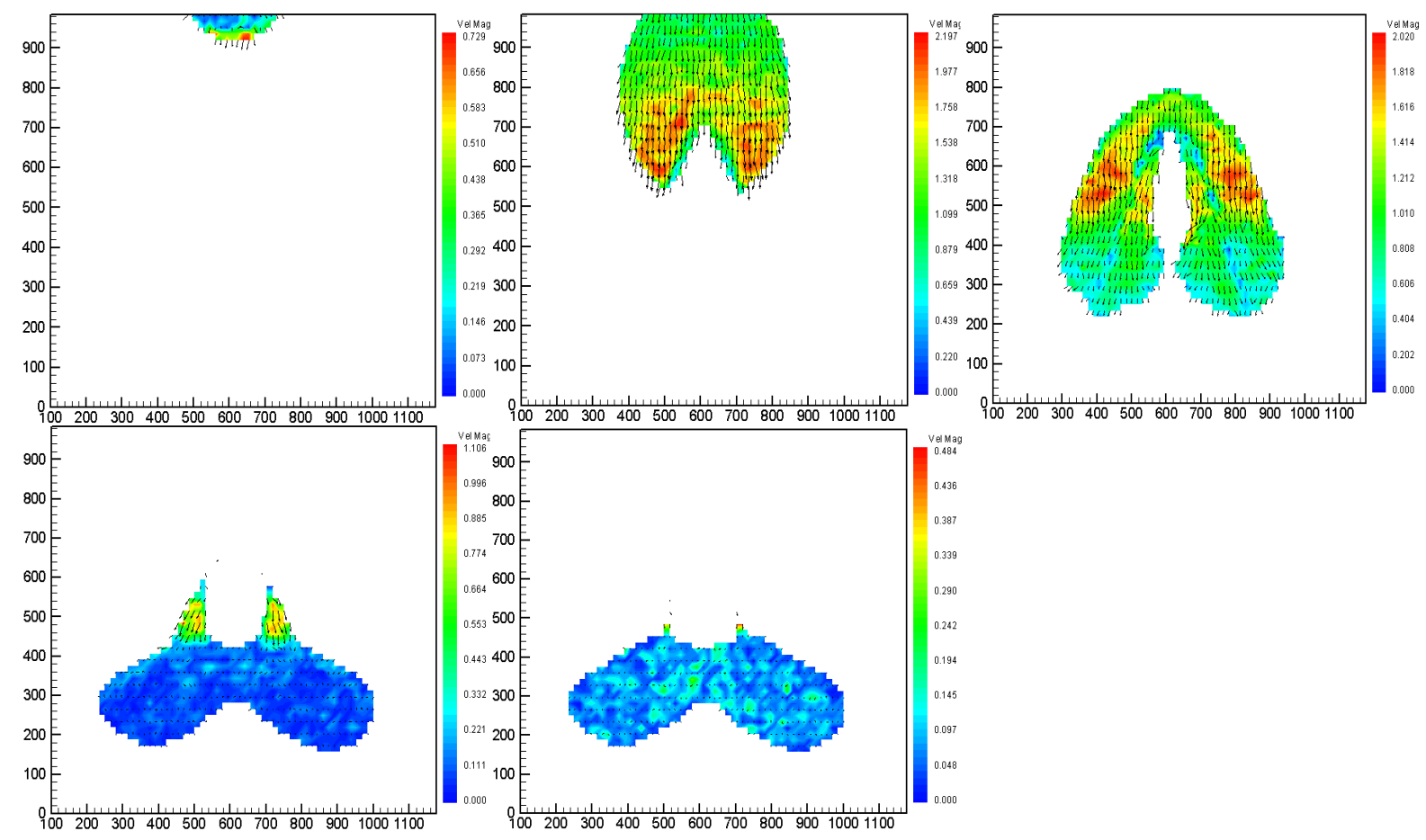

Figure 10: Photographs from the experiment (panel a) and the velocity fields of PIV measurement (panel b) at several times. The color in panel (b) indicates the value of the surface velocity. These experimental results are well documented in the PhD thesis of Chiou [2006]. All experiments have been performed in the laboratory at the Technical University of Darmstadt 


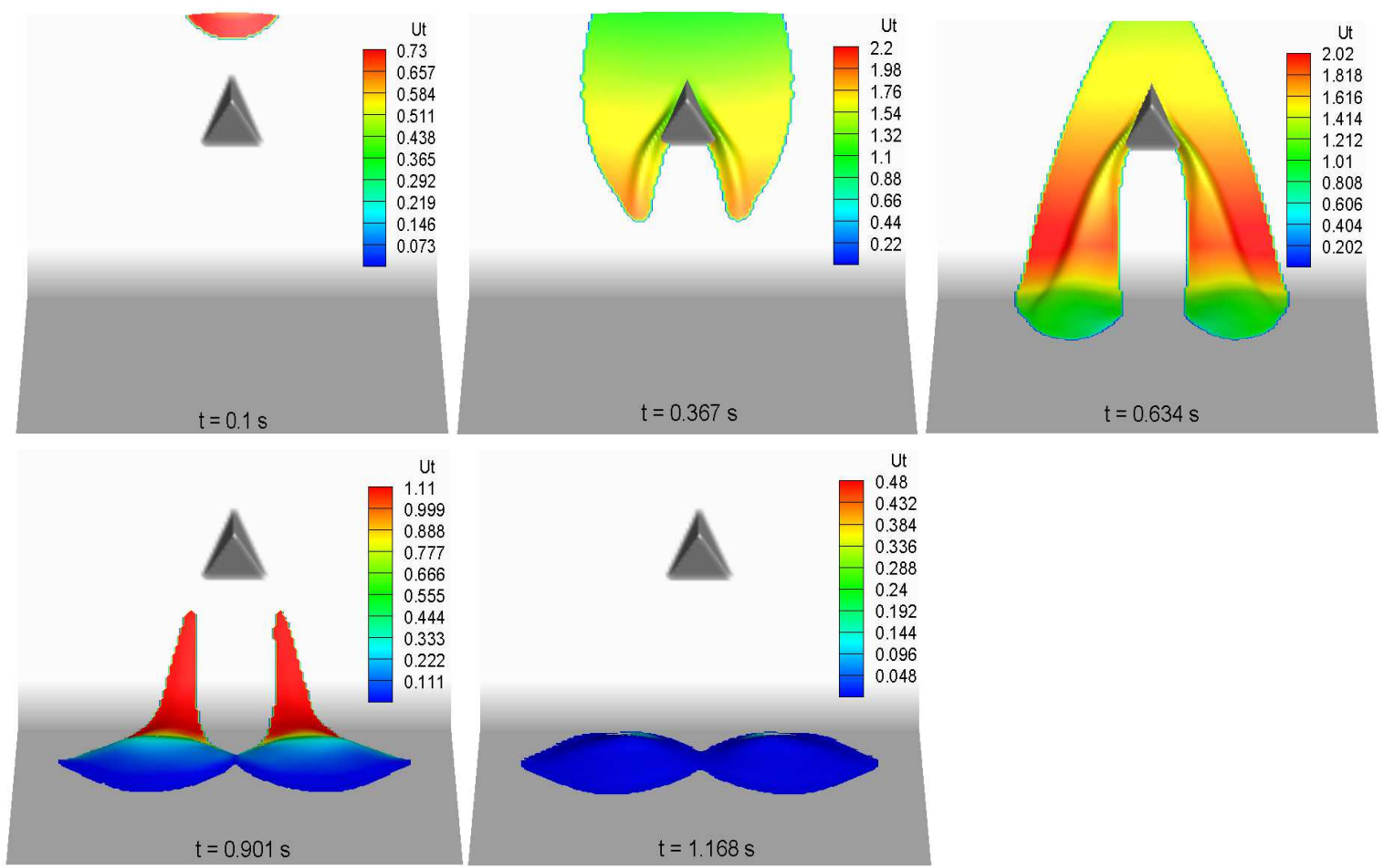

Figure 11: Numerical prediction of velocity field of dry granular flow. The velocity field is demonstrated by the color. 

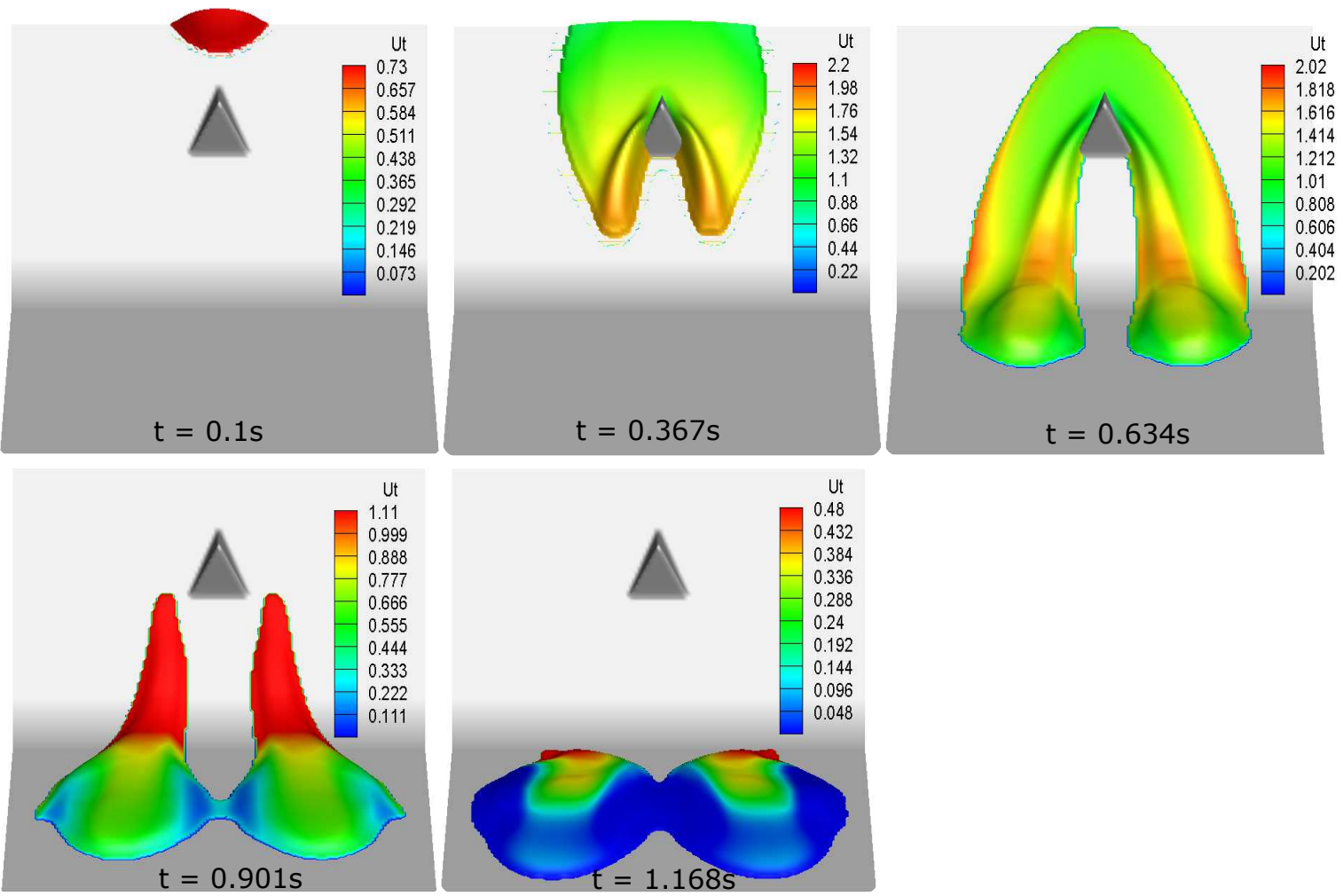

Figure 12: Numerical prediction of the liquid-grain depth profile. The velocity field is demonstrated by the color. 


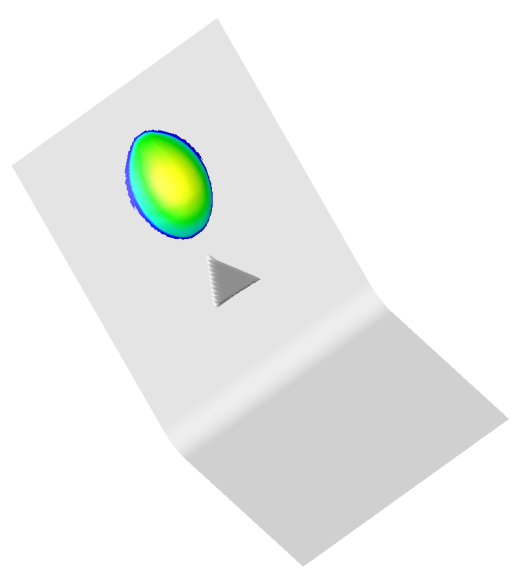

(a) $\mathrm{t}=0.1 \mathrm{~s}$

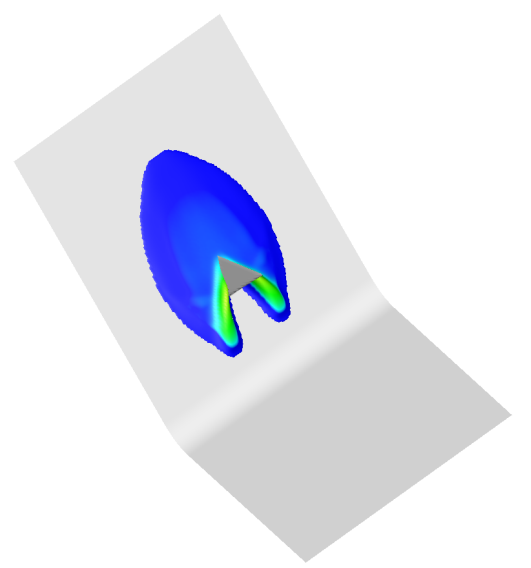

(b) $\mathrm{t}=0.367 \mathrm{~s}$

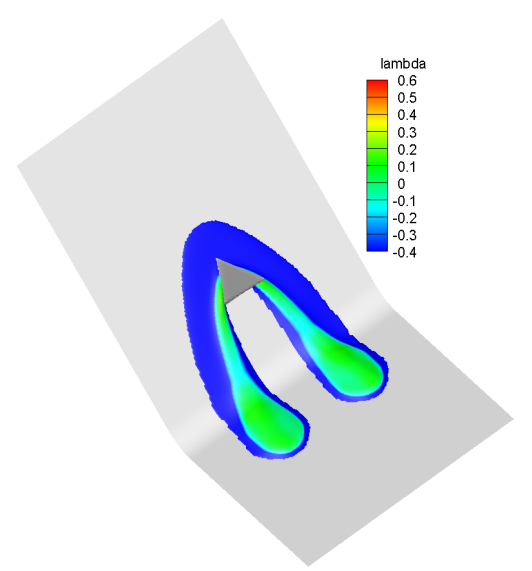

(c) $\mathrm{t}=0.634 \mathrm{~s}$

Figure 13: Three dimensional geometries of liquid-grain mixture flows at times $t=0.1 \mathrm{~s}, 0.367 \mathrm{~s}$, and $0.634 \mathrm{~s}$, consecutively. The color indicates the distribution of the dimensionless basal pore fluid pressure $\lambda=p_{\text {bed }} /(\rho g h \cos \zeta)$. 